\title{
Wavelets on Fractals
}

\section{Dorin E. Dutkay and Palle E. T. Jorgensen}

\author{
Dedicated to the memory of Gert Kjargaard Pedersen
}

\begin{abstract}
We show that there are Hilbert spaces constructed from the Hausdorff measures $\mathcal{H}^{s}$ on the real line $\mathbb{R}$ with $0<s<1$ which admit multiresolution wavelets. For the case of the middle-third Cantor set $\mathbf{C} \subset[0,1]$, the Hilbert space is a separable subspace of $L^{2}\left(\mathbb{R},(d x)^{s}\right)$ where $s=\log _{3}(2)$. While we develop the general theory of multiresolutions in fractal Hilbert spaces, the emphasis is on the case of scale 3 which covers the traditional Cantor set C. Introducing

$$
\psi_{1}(x)=\sqrt{2} \chi_{\mathbf{C}}(3 x-1) \quad \text { and } \quad \psi_{2}(x)=\chi_{\mathbf{C}}(3 x)-\chi_{\mathbf{C}}(3 x-2)
$$

we first describe the subspace in $L^{2}\left(\mathbb{R},(d x)^{s}\right)$ which has the following family as an orthonormal basis (ONB):

$$
\psi_{i, j, k}(x)=2^{j / 2} \psi_{i}\left(3^{j} x-k\right),
$$

where $i=1,2, j, k \in \mathbb{Z}$.

Since the affine iteration systems of Cantor type arise from a certain algorithm in $\mathbb{R}^{d}$ which leaves gaps at each step, our wavelet bases are in a sense gap-filling constructions.
\end{abstract}

\section{Introduction}

The paper has three interrelated themes: (1) construction of wavelet bases in separable Hilbert spaces built on affine fractals and Hausdorff measure; (2) approximation of the corresponding wavelet scaling functions, using the cascading approximation algorithm; and (3) an associated spectral theoretic analysis of a transfer operator, often called the Ruelle operator.

2000 Mathematics Subject Classification: 41A15, 42A16, 42A65, 42C40, 43A65, 46L60, 47D25, 46L45.

Keywords: Hausdorff measure, Cantor sets, iterated function systems (IFS), fractal, wavelets, Hilbert space, unitary operators, orthonormal basis (ONB), spectrum, transfer operator, cascade approximation, scaling, translation. 
There are surprises when our results are compared to what is known for the traditional multiresolution approach for $L^{2}\left(\mathbb{R}^{d}\right)$, and even when compared to known results for special classes of affine fractals.

Some comments on (1)-(3): Due to earlier work by Jorgensen and Pedersen [16] and Strichartz et al [23], it is known that a subclass of the affine fractals admits Fourier duality. Affine fractals arise from the specification of an expansive matrix, and a finite set of translations. The fractal $X$ itself then arises from this data and an iteration 'in the small' of the corresponding affine maps. Let $L=L(X)$ be the associated iteration 'in the large'. We say that $(X, L)$ is a Fourier duality, if an orthonormal basis on $X$ may be built from the frequencies in $L$. While it is known that, if $X$ is the middle third Cantor set, then there is no $L$ which makes a duality pair, we show that nonetheless, every affine fractal admits an orthonormal wavelet basis. In our discussion of wavelets, we start with the middle third Cantor set; and we then pass on to the general affine fractals.

As for the approximation issues in (2), we know that for $L^{2}\left(\mathbb{R}^{d}\right)$, there is a rich family of wavelet filters which yield cascade approximation. This family of filters is much more restricted for the fractals: Our results for the affine fractals even offer a certain dichotomy (Theorem 6.2): If the cascades do not converge in the Hilbert space, then the terms in the cascading approximation sequence are typically orthogonal, and thus very far from being convergent.

Our analysis of (1)-(2) is based on spectral theory of the associated transfer operator, and we show in the second half of the paper (starting with Section 4) how this spectral theory differs in the three cases, the standard $L^{2}\left(\mathbb{R}^{d}\right)$-wavelets, and the special duality fractals versus the general class of affine fractals.

Our proofs depend on ideas from geometric measure theory, and from earlier papers on harmonic analysis of affine fractals. While some of this material is in the literature, it isn't available precisely in the form we need it here. In any case, it is difficult for readers to locate without first having a brief overview. So we include a minimum amount of facts from the literature for the benefit of readers. We hope thereby to bridge the diverse fields, fractals, Hilbert space, wavelets, approximation, and harmonic analysis.

We develop the theory of multiresolutions in the context of Hausdorff measure of fractional dimension between 0 and 1 . While our fractal wavelet theory has points of similarity that it shares with the standard case of Lebesgue measure on the line, there are also sharp contrasts. These are stated in our main result, a dichotomy theorem. The first section is the case of the middle-third Cantor set. This is followed by a review of the essentials on Hausdorff measure. The remaining sections of the paper cover multiresolutions in the general context of affine iterated function systems. 
It is well known that the Hilbert spaces $L^{2}(\mathbb{R})$ has a rich family of orthonormal bases of the following form:

$$
\psi_{j, k}(x)=2^{j / 2} \psi\left(2^{j} x-k\right), \quad j, k \in \mathbb{Z},
$$

where $\psi$ is a single function $\in L^{2}(\mathbb{R})$, with

$$
\|\psi\|_{2}=\left(\int_{\mathbb{R}}|\psi(x)|^{2} d x\right)^{1 / 2}=1,
$$

and the integration refers to the usual Lebesgue measure on $\mathbb{R}$. Take for example

$$
\psi(x)=\chi_{I}(2 x)-\chi_{I}(2 x-1)
$$

where $I=[0,1]$ is the unit interval. Clearly $I$ satisfies

$$
2 I=I \cup(I+1) .
$$

The Cantor subset $\mathbf{C} \subset I$ satisfies

$$
3 \mathbf{C}=\mathbf{C} \cup(\mathbf{C}+2)
$$

and its indicator function $\varphi_{\mathbf{C}}:=\chi_{\mathbf{C}}$ satisfies

$$
\varphi_{\mathbf{C}}\left(\frac{x}{3}\right)=\varphi_{\mathbf{C}}(x)+\varphi_{\mathbf{C}}(x-2) .
$$

Since both constructions, the first one for the Lebesgue measure, and the second one for the Hausdorff version $(d x)^{s}$, arise from scaling and subdivision, it seems reasonable to expect multiresolution wavelets also in Hilbert spaces constructed on the scaled Hausdorff measures $\mathcal{H}^{s}$ which are basic for the kind of iterated function systems which give Cantor constructions built on scaling and translations by lattices. We show this to be the case, but there are still striking differences between the two settings, and we spell out some of them after first developing the theory in the case of the middle-third Cantor construction.

While there are other wavelet approaches to fractals in the literature, for example [13], [14], and [21], there is in fact no overlap with this work, since the previous papers deal with wavelets on the fractal itself, while the present paper deals with wavelets on an enlarged fractal (actually a fractal measure), allowing a structure closer to a standard multiresolution analysis (MRA).

The practical applications are to fractals arising in physics and in symbolic dynamical systems from theoretical computer science, see e.g., [2] [19], [24]. There is already a considerable body of work on harmonic analysis on fractals, see for example [22], [11], [10], [23], and [16]. Much of it is based on subdivision techniques, and algorithms which use cascade constructions, but so far we have not seen direct wavelet algorithms and wavelet analysis for fractals. 
In section 2, we recall some facts about Hausdorff measure $\mathcal{H}^{s}$, Hausdorff dimension, and Hausdorff distance. They will be needed in the Hilbert space we build on $\mathcal{H}^{s}$. It is a natural separable subspace of the full $\mathcal{H}^{s}$-Hilbert space, and it is built up from the algebra of $\mathbb{Z}$-translations (additive), and $N$-adic scaling (multiplicative), where $N$ is fixed. We then turn to the cascade approximation for the scaling function $\varphi$ defined by the usual $1 / N$ subdivision. We prove a theorem for the case $0<s<1$ which stands in sharp contrast to the traditional and more familiar case $s=1$ of Daubechies et. al.; i.e., the case of the Hilbert space $L^{2}(\mathbb{R})$ based on Lebesgue measure $d x$ on $\mathbb{R}$ : The scaling equation is then

$$
\varphi(x)=\sqrt{N} \sum_{k \in \mathbb{Z}} a_{k} \varphi(N x-k)
$$

with the masking coefficients $a_{k}$ satisfying the usual two axioms

$$
\sum_{k \in \mathbb{Z}} a_{k}=\sqrt{N}, \text { and } \sum_{k \in \mathbb{Z}} \bar{a}_{k} a_{k+N \ell}=\delta_{\ell, 0}, \ell \in \mathbb{Z} .
$$

Motivated by the expression on the right hand side in (1.4), we define the wavelet subdivision operator $M$ by

$$
(M f)(x):=\sqrt{N} \sum_{k \in \mathbb{Z}} a_{k} f(N x-k), \quad f \in L^{2}(\mathbb{R}) ;
$$

and note that its properties depend on the specifications in (1.5).

Simple conditions are known for when the limit

$$
\lim _{n \rightarrow \infty} M^{n} \chi_{I}=\varphi
$$

exists in $L^{2}(\mathbb{R})$, see [7], chapter 5. Then $\varphi$ (when it exists) solves (1.4), and there is an easy formula for building functions $\psi_{1}, \ldots, \psi_{N-1}$ in $L^{2}(\mathbb{R})$ from $\varphi$ such that

$$
\left\{N^{\frac{k}{2}} \psi_{i}\left(N^{k} x-\ell\right) \mid 1 \leq i<N, k, \ell \in \mathbb{Z}\right\}
$$

is an orthonormal basis $(\mathrm{ONB})$ in $L^{2}(\mathbb{R})$. If $N=2$, a formula for $\psi$ is

$$
\psi(x)=\sqrt{2} \sum_{k \in \mathbb{Z}}(-1)^{k} \bar{a}_{1-k} \varphi(2 x-k) .
$$

In general when $N \geq 2$, the functions $\psi_{1}, \ldots, \psi_{N-1}$ may result from the solution to a simple matrix completion problem; see [20], [5] and [6] for details. In the case of Hausdorff measure $\mathcal{H}^{s}(0<s<1, s$ depending on the scaling number $N$ ), the analogous matrix completion is still fairly simple. 
A main question (non-trivial) is now that of solving the analogue of (1.4), but in the $\mathcal{H}^{s}$-Hilbert space. The biggest differences concern the changes in (1.5) and (1.7) when $0<s<1$. It turns out in the fractal cases that there are then many fewer admissible solutions than those suggested by analogy with (1.5). We summarize the situation in Sections 4-6, where our main result takes the form of a dichotomy theorem; the solutions to the $\mathcal{H}^{s}$-convergence question are isolated within a larger family of masking coefficients analogous to (1.5). There is further a new set of orthogonality conditions entering the analysis when $0<s<1$, which are not present in the more familiar case of $s=1$.

We interpret the wavelet filters as functions $m_{0}$ on the torus $\mathbb{T}$. If the scaling number $N$ is given, following [15], we introduce the wavelet-transfer operator

$$
\left(R_{m_{0}} f\right)(z):=\frac{1}{N} \sum_{w^{N}=z}\left|m_{0}(w)\right|^{2} f(w), \quad \text { for } f \in C(\mathbb{T}), \text { and } z \in \mathbb{T} .
$$

Our dichotomy for wavelets will be explained in terms of the spectral properties of $R_{m_{0}}$, also called the Ruelle operator. For simplicity, we introduce the normalization $R_{m_{0}}(\hat{1})=\hat{1}$, where $\hat{1}$ denotes the constant function 1 on $\mathbb{T}$. A probability measure $\nu$ on $\mathbb{T}$ is said to be invariant if $\nu R_{m_{0}}=\nu$. Equivalently,

$$
\int_{\mathbb{T}} R_{m_{0}}(f) d \nu=\int_{\mathbb{T}} f d \nu, \quad \text { for all } f \in C(\mathbb{T}),
$$

or

$$
\nu\left(R_{m_{0}}(f)\right)=\nu(f) .
$$

We introduce a notion of $\left(m_{0}, N\right)$-cycles for (1.10) which explains the solutions $\nu \in M_{1}(\mathbb{T})$ to (1.11). In our setting, the dichotomy boils down to two cases for $\nu$ :

(i) $\nu=\delta_{1}$ (the Dirac mass at $z=1$ ), or

(ii) some $\nu$ is a singular measure on $\mathbb{T}$ with full support.

In the first case $(i)$, the Hilbert space is $L^{2}(\mathbb{R})$; i.e., that of the standard wavelets; and in the second case (ii), the Hilbert space is built from Hausdorff measure $\mathcal{H}^{s}, 0<s<1$.

We begin the discussion with $N=3$ and $s=\log _{3}(2)$.

Definition 1.1 We define $\mathcal{R}$ to be the set of all real numbers that have a base 3 expansion containing only finitely many ones. It is an inflated version of $\mathbf{C}$ :

$$
\mathcal{R}:=\left\{\begin{array}{c|c}
\sum_{k=-m}^{\infty} a_{k} 3^{-k} \mid \begin{array}{c}
m \in \mathbb{Z}, a_{k} \in\{0,1,2\} \text { for all } k \in \mathbb{Z}, \\
a_{k} \neq 1 \text { for all but finitely many indices } k .
\end{array}
\end{array}\right\}
$$


For the fractal cases, $0<s<1$, the factor $\sqrt{N}$ in equations (1.4)-(1.6) will be different, see details below. Similarly the factor $N^{\frac{k}{2}}$ in (1.8) changes: With scaling number $N$ and with $p$ subdivisions, the ONB corresponding to $(1.8)$ in $L^{2}\left(\mathcal{R}, \mathcal{H}^{s}\right), s=\log _{N}(p)$ is $\left\{p^{\frac{k}{2}} \psi_{i}\left(N^{k} x-\ell\right)\right\}$. However, the geometric properties of the cascade approximation to the scaling function change completely as the Hausdorff dimension moves from $s=1$ to the open interval $0<s<1$. This will be spelled out in the last four sections of the paper.

\section{The Hausdorff Measure}

Returning to the middle-third Cantor set $\mathbf{C}=\mathbf{C}_{3}$; i.e., $N=3$ and $p=2$, here are some elementary properties of $\mathcal{R}$ :

Proposition 2.1 (The middle-third Cantor set) The set $\mathcal{R}$ has the following properties:

(i) Invariance under triadic translation:

$$
\mathcal{R}+\frac{k}{3^{n}}=\mathcal{R}, \quad(k, n \in \mathbb{Z}) .
$$

(ii) Invariance under dilation by 3 :

$$
3^{n} \mathcal{R}=\mathcal{R}, \quad(n \in \mathbb{Z}) .
$$

(iii) The middle-third Cantor set $\mathbf{C}$ is contained in $\mathcal{R}$ and moreover it covers $\mathcal{R}$ by translations and dilations:

$$
\mathcal{R}=\bigcup_{n \in \mathbb{Z}} \bigcup_{k \in \mathbb{Z}} 3^{-n}(\mathbf{C}+k)
$$

Proof. (i) Any triadic number $t=\frac{k_{0}}{3^{n} 0}$ with $k_{0}, n_{0} \in \mathbb{Z}, k_{0} \geq 0$, has a finite expansion in base 3 :

$$
t=\sum_{k=-m}^{m} t_{k} 3^{-k}, \quad t_{k} \in\{0,1,2\} .
$$

Take $x \in \mathcal{R}$. Then $x$ has a finite number of ones in its expansion so the same affirmation will be true for $x+t$.

(ii) is clear: multiplication by 3 means a shift in the base 3 expansion.

(iii) Since

$$
\mathbf{C}=\left\{\sum_{k=1}^{\infty} a_{k} 3^{-k} \mid a_{k} \in\{0,2\}\right\},
$$

it is obvious that $\mathbf{C} \subset \mathcal{R}$. 
The inclusion " $\supset$ " follows from (i) and (ii). Now take

$$
x \in \mathcal{R}, \quad x=\sum_{k=-m}^{\infty} a_{k} 3^{-k},
$$

only finitely many $a_{k}$ are equal to 1 . Then

Let $\ell_{0}$ be the last index for which $a_{l_{0}}=1$, and take $k_{0}:=\max \left\{n_{0}, \ell_{0}\right\}$.

$$
x \in 3^{-k_{0}}\left(\mathbf{C}+\sum_{k=-m}^{k_{0}} a_{k} 3^{-k+k_{0}}\right)
$$

and this shows that the other inclusion is also true.

Remark 2.2 $\mathcal{R}$ has Lebesgue measure 0. Indeed, this follows from proposition 2 (iii), because $\mathbf{C}$ has Lebesgue measure 0 , and so do all the sets $3^{-n}(\mathbf{C}+k)$ with $n, k \in \mathbb{Z}$.

Even though some of the properties that we need for the Hausdorff measure, for fractals, and for iterated function systems (IFS) are known, we found that the material is wildly scattered throughout the literature; and to increase readability we have included some highpoints from these areas. This should also help bring out the contrast between the traditional MRAanalysis, and the present more stochastic approach.

Next we define a measure on $\mathcal{R}$. It is the restriction of the Hausdorff measure $\mathcal{H}^{s}$ with $s=\log _{3}(2)$ to $\mathcal{R}$.

We recall some background on the Hausdorff measures from [9]:

For a subset $E$ of $\mathbb{R}, s>0$, and $\delta>0$, define

$$
\mathcal{H}_{\delta}^{s}(E):=\inf \left\{\sum_{i=1}^{\infty}\left|U_{i}\right|^{s}\left|\bigcup_{i=1}^{\infty} U_{i} \supset E,\right| U_{i} \mid<\delta\right\}
$$

where $|U|=\sup \{|x-y| \mid x, y \in U\}$ (the diameter of $U$ ).

It is known that $\mathcal{H}_{\delta}^{s}$ is an outer measure on $\mathbb{R}$.

Define

$$
\mathcal{H}^{s}(E)=\lim _{\delta \rightarrow 0} \mathcal{H}_{\delta}^{s}(E)=\sup _{\delta>0} \mathcal{H}_{\delta}^{s}(E)
$$

Then verify that $\mathcal{H}^{s}$ is also an outer measure. By the Caratheodory construction [9], if we restrict $\mathcal{H}^{s}$ to the $\sigma$-field of $\mathcal{H}^{s}$-measurable sets, we get a measure called the Hausdorff measure. 


\section{Proposition 2.3}

(i) All Borel sets are measurable.

(ii) [Inner regularity $]$ Any $\mathcal{H}^{s}$-measurable set of finite $\mathcal{H}^{s}$-measure contains a $F_{\sigma}$-set of equal $\mathcal{H}^{s}$-measure.

(iii) If $E \subset \mathcal{R}$ then there is a $G_{\delta^{-}}$set containing $E$ and of the same $\mathcal{H}^{s}$ measure.

(iv) For $s<1$ and $G$ open, $\mathcal{H}^{s}(G)=\infty$ (the measure is not regular from above).

(v) Translation invariance: For any $\mathcal{H}^{s}$-measurable set $E$, and any $t \in \mathcal{R}$, $E+t$ is $\mathcal{H}^{s}$-measurable, and

$$
\mathcal{H}^{s}(E)=\mathcal{H}^{s}(E+t)
$$

(vi) For any $\mathcal{H}^{s}$-measurable set $E$ and any $c>0, c E$ is $\mathcal{H}^{s}$-measurable and

$$
\mathcal{H}^{s}(c E)=c^{s} \mathcal{H}^{s}(E)
$$

Consider now $\mathcal{H}^{s}$ with $s=\log _{3} 2$ restricted to the $\mathcal{H}^{s}$-measurable subsets of $\mathcal{R}$. We will keep the notation $\mathcal{H}^{s}$ for the restriction.

\section{Proposition 2.4}

(i) If $E \subset \mathcal{R}$ is an $\mathcal{H}^{s}$-measurable set and $t=\frac{l_{0}}{3^{p_{0}}}$ is a triadic number, then $E+t \subset R$ is $\mathcal{H}^{s}$-measurable and

$$
\mathcal{H}^{s}(E)=\mathcal{H}^{s}(E+t)
$$

(ii) If $E \subset \mathcal{R}$ is an $\mathcal{H}^{s}$-measurable set then $3 E \subset \mathcal{R}$ is $\mathcal{H}^{s}$-measurable, and

$$
\mathcal{H}^{s}(3 E)=2 \mathcal{H}^{s}(E)
$$

(iii) If $f \in L^{1}\left(\mathcal{R}, \mathcal{H}^{s}\right)$ then the function on $\mathcal{R}, x \rightarrow f\left(\frac{x}{3}\right)$ is also in $L^{1}\left(\mathcal{R}, \mathcal{H}^{s}\right)$ and

$$
\int_{\mathcal{R}} f(x) d \mathcal{H}^{s}(x)=\frac{1}{2} \int_{\mathcal{R}} f\left(\frac{x}{3}\right) d \mathcal{H}^{s}(x) .
$$

(iv) $\mathcal{H}^{s}(\mathbf{C})=1$, where $\mathbf{C}$ is the middle-third Cantor set.

Proof. (i) and (ii) are direct consequences of propositions 2.3 and 2.4.

(iii) follows from (ii) when $f$ is a characteristic function of an $\mathcal{H}^{s}$-measurable set. Then, for arbitrary $f$, the formula can be obtained by approximations by simple functions.

(iv) See [9, theorem 1.14]. 
Remark 2.5 The measure $\mathcal{H}^{s}$ on $\mathcal{R}$ is still non-regular from above. All open sets in $\mathcal{R}$ still have infinite measure.

To see this, we show that $\mathcal{H}^{s}(I)=\infty$, where $I=(0,1) \cap \mathcal{R}$. Indeed $I \supset \mathbf{C}$, so $\mathcal{H}^{s}(I) \geq 1$. Also, observe that $3 I=I \cup(I+1) \cup(I+2)$ disjoint union (we neglect some points that have $\mathcal{H}^{s}$-measure 0 .). Therefore, with propositions 2.4 (i) and (ii), we obtain

$$
2 \mathcal{H}^{s}(I)=3 \mathcal{H}^{s}(I)
$$

so $\mathcal{H}^{s}(I)$ is either 0 or $\infty$. 0 cannot be from the previous argument, hence it must be $\infty$.

By scalings and translations, it can be proved that $\mathcal{H}^{s}((a, b) \cap \mathcal{R})=\infty$ for any interval $(a, b)$.

Since all open subsets of $\mathcal{R}$ have measure $\infty$ it follows that no nonzero continuous function on $\mathbb{R}$ is integrable! (Just take $f^{-1}((a, b))$ for some interval that doesn't contain 0 and intersects the range.)

Definition 2.6 We denote by $H$ the Hilbert space

$$
H:=L^{2}\left(\mathcal{R}, \mathcal{H}^{s}\right)
$$

The linear operator $T$ on $H$ defined by

$$
T f(x)=f(x-1), \quad(f \in H, x \in \mathcal{R}),
$$

is called the translation operator. The linear operator $U$ on $H$ defined by

$$
U f(x)=\frac{1}{\sqrt{2}} f\left(\frac{x}{3}\right), \quad(f \in H, x \in \mathcal{R})
$$

is called the dilation operator.

From Proposition 2.4, and using some simple computations, we obtain the following proposition:

\section{Proposition 2.7}

(i) $T$ and $U$ are unitary operators.

(ii) $U T U^{-1}=T^{3}$.

Denote by $\varphi=\chi_{\mathbf{C}}$, the characteristic function of the Cantor set $C$. We prove that $\varphi$ satisfies all the properties of a scaling vector. 
Proposition 2.8 The following hold:

(i) [The scaling equation] $U \varphi=\frac{1}{\sqrt{2}}\left(\varphi+T^{2} \varphi\right)$.

(ii) [Orthogonality of the translates] $\left\langle T^{k} \varphi \mid \varphi\right\rangle=\delta_{k},(k \in \mathbb{Z})$.

(iii) [Cyclicity] $\overline{\operatorname{span}}\left\{U^{n} T^{k} \varphi \mid n \in \mathbb{Z}, k \in \mathbb{Z}\right\}=H$.

\section{Proof.}

(i) $U \varphi=\frac{1}{\sqrt{2}} \chi_{3 \mathbf{C}}, T^{2} \varphi=\chi_{\mathbf{C}+2}$, but $3 \mathbf{C}=\mathbf{C} \bigcup(\mathbf{C}+2)$, so (i) follows.

(ii) $T^{k} \varphi=\chi_{\mathbf{C}+k}$ so $T^{k} \varphi$ and $\varphi$ are disjointly supported for $k \neq 0$. For $k=0$, $\langle\varphi \mid \varphi\rangle=\int_{\mathcal{R}} \chi_{\mathbf{C}} d \mathcal{H}^{s}=1$, by proposition 2.8 (iv).

(iii) First take $E \subset R$ measurable and with $\mathcal{H}^{s}(E)<\infty$. We want to approximate $\chi_{E}$ by linear combinations of functions of the form $U^{n} T^{k} \varphi$.

Note also that

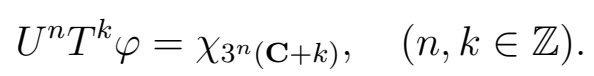

With proposition 2.4(iii), and partitioning $E$ if necessary, we may assume that $E$ is contained in a set of the form $3^{n^{0}}\left(\mathbf{C}+k_{0}\right)$. Applying dilations and translations we may further assume that $E \subset \mathbf{C}$

Define

$$
\mathcal{V}:=\left\{C_{n, a_{n}, \ldots, a_{1}}:=3^{-n} \mathbf{C}+\sum_{k=1}^{n} a_{k} 3^{-k} \mid a_{k} \in\{0,2\} n \geq 1\right\} .
$$

This family $\mathcal{V}$ is a Vitali class for $E$; i.e., for each $x \in E$ and each $\delta>0$, there is a $U \in \mathcal{V}$ with $x \in U$, and $0<|U| \leq \delta$.

Indeed, we see that for all $n \geq 1$ :

$$
\bigcup_{a_{1}, \ldots, a_{n} \in\{0,2\}} C_{n, a_{n}, \ldots, a_{1}}=\mathbf{C}
$$

Also using proposition 2.8

$$
\mathcal{H}^{s}\left(C_{n, a_{n}, \ldots, a_{1}}\right)=\mathcal{H}^{s}\left(3^{-n} \mathbf{C}\right)=\left(3^{-n}\right)^{s} \mathcal{H}^{s}(\mathbf{C})=2^{-n}=\left|C_{n, a_{n}, \ldots, a_{1}}\right|^{s} .
$$

We conclude that $\mathcal{V}$ is indeed a Vitali class for any subset $E$ of $\mathbf{C}$.

Then, by Vitali's covering theorem (see [9, theorem 1.10]) for a fixed $\varepsilon>0$, there exists a finite or countable disjoint sequence of sets $\left\{U_{i}\right\}$ from $\mathcal{V}$ such that either $\sum\left|U_{i}\right|^{s}=\infty$, or $\mathcal{H}^{s}\left(E \backslash \bigcup U_{i}\right)=0$, and also

$$
\mathcal{H}^{s}(E)<\sum_{i}\left|U_{i}\right|^{s}+\varepsilon
$$


Since the sets $U_{i}$ are mutually disjoint and contained in $\mathbf{C}$, and using (i) in $(2.11)$, it follows that

$$
\sum_{i}\left|U_{i}\right|^{s}=\sum_{i} \mathcal{H}^{s}\left(U_{i}\right)=\mathcal{H}^{s}\left(\bigcup_{i} U_{i}\right) \leq \mathcal{H}^{s}(\mathbf{C})=1
$$

Therefore the other variant must be true:

$$
\mathcal{H}^{s}(E \backslash U)=0 \quad \text { with } U:=\bigcup_{i} U_{i}
$$

On the other hand

$$
\begin{aligned}
\mathcal{H}^{s}(U \backslash E) & =\mathcal{H}^{s}(U)-\mathcal{H}^{s}(U \cap E)=\mathcal{H}^{s}(U)-\left(\mathcal{H}^{s}(E)-\mathcal{H}^{s}(E \backslash U)\right) \\
& =\mathcal{H}^{s}(U)-\mathcal{H}^{s}(E)=\sum_{i} \mathcal{H}^{s}\left(U_{i}\right)-\mathcal{H}^{s}(E) \\
& =\sum_{i}\left|U_{i}\right|^{s}-\mathcal{H}^{s}(E)<\varepsilon .
\end{aligned}
$$

Also, observe that

$$
C_{n, a_{n}, \ldots, a_{1}}=3^{-n}\left(\mathbf{C}+\sum_{k=1}^{n} a_{k} 3^{n-k}\right),
$$

so by $(2.10), \chi_{C_{n, a_{n}, \ldots, a_{1}}}=U^{-n} T^{l} \varphi$ with $l=\sum_{k=1}^{n} a_{k} 3^{n-k}$.

Therefore we see that all measurable sets $E \subset \mathcal{R}$ with $\mathcal{H}^{s}(E)<\infty$ are in the span of $\left\{U^{n} T^{k} \varphi \mid n, k \in \mathbb{Z}\right\}$. Since all integrable functions $f \in H$ can be approximated by simple functions, it follows that

$$
H=\overline{\operatorname{span}}\left\{U^{n} T^{k} \varphi \mid n, k \in \mathbb{Z}\right\} .
$$

\section{Iterated Function Systems (IFS) and gap-filling wavelets}

The middle-third Cantor set $\mathbf{C}$ of Section 1 is a special case of an Iterated Function System (IFS). It falls in the subclass of the IFSs which are called affine. Specifically, let $d \in \mathbb{Z}_{+}$, and let $A$ be a $d \times d$ matrix of $\mathbb{Z}$. Suppose that the eigenvalues $\lambda_{i}$ of $A$ satisfy $\left|\lambda_{i}\right|>1$. Set $N:=|\operatorname{det} A|$. These matrices are called expansive. Then note that the quotient group $\mathbb{Z}^{d} / A\left(\mathbb{Z}^{d}\right)$ is of order $N$. A subset $\mathcal{D} \subset \mathbb{Z}^{d}$ is said to represent the $A$-residues if the natural quotient mapping

$$
\gamma: \mathbb{Z}^{d} \rightarrow \mathbb{Z}^{d} / A\left(\mathbb{Z}^{d}\right)
$$

restricts to a bijection $\gamma_{\mathcal{D}}$ of $\mathcal{D}$ onto $\mathbb{Z}^{d} / A\left(\mathbb{Z}^{d}\right)$. 
For example, if $d=1$, and $A=3$, then we may take either one of the two sets $\{0,1,2\}$ or $\{0,1,-1\}$ as $\mathcal{D}$. The IFSs which we shall look at will be constructed from finite subsets $\mathcal{S} \subset \mathbb{Z}^{d}$ which represent the $A$-residues for some given expansive matrix $A$. If $(A, \mathcal{S})$ is a pair with these properties, define the maps

$$
\sigma_{s}(x):=A^{-1}(x+s), s \in \mathcal{S}, x \in \mathbb{R}^{d} .
$$

Using a theorem of Hutchinson [12], we conclude that there is a unique measure $\mu=\mu_{(A, \mathcal{S})}$ with compact support $\mathbf{C}=\mathbf{C}_{(A, \mathcal{S})}$ on $\mathbb{R}^{d}$ such that

$$
\mu=\frac{1}{\#(\mathcal{S})} \sum_{s \in \mathcal{S}} \mu \circ \sigma_{s}^{-1},
$$

or equivalently

$$
\int f(x) d \mu(x)=\frac{1}{\#(\mathcal{S})} \sum_{s \in \mathcal{S}} \int f\left(\sigma_{s}(x)\right) d \mu(x) .
$$

The quotient mapping

$$
\gamma: \mathbb{R}^{d} \rightarrow \mathbb{T}^{d}:=\mathbb{R}^{d} / \mathbb{Z}^{d}
$$

restricts to map $\mathbf{C}$ bijectively onto a compact subset of $\mathbb{T}^{d}$. The Hausdorff dimension $h$ of $\mu$ and of the support $\mathbf{C}$ is

$$
h=\frac{\log \#(\mathcal{S})}{\log N} .
$$

The system $(\mathbf{C}, \mu)$ is called a Hutchinson pair, see lemma 3.5.

If $d=1$, we will look at two examples: (i) $(A, \mathcal{S})=(3,\{0,2\})$ which is the middle-third Cantor set $\mathbf{C}$ in Section 1, and (ii) $(A, \mathcal{S})=(4,\{0,2\})$ which is the corresponding construction, but starting with a subdivision of the unit interval $I$ into 4 parts, and in each step of the iteration omitting the second and the fourth quarter interval. As noted, then

$$
h_{(i)}=\log _{3}(2)=\frac{\log 2}{\log 3}, \quad \text { and } \quad h_{(i i)}=\frac{1}{2}
$$

for more details, see [16].

Since the arguments from proposition 2.4 and 2.8 generalize, we will only sketch the general statements of results for the affine IFSs, those based on pairs $(A, \mathcal{S})$ in $\mathbb{R}^{d}$ where the matrix $A$ and the subset $\mathcal{S} \subset \mathbb{Z}^{d}$ satisfy the stated conditions. The number $h$ will be $h=\frac{\log (\#(\mathcal{S}))}{\log |\operatorname{det} A|}$; i.e., the Hausdorff 
dimension of the measure $\mu$, and its support $\mathbf{C}$ which are determined from the given pair $(A, \mathcal{S})$. We will then be working with the corresponding Hausdorff measure $\mathcal{H}^{h}$, but now as a measure defined on subsets of $\mathbb{R}^{d}$. The facts from Section 2 apply also to this more general case in $\mathbb{R}^{d}$, for example, property (1.2) for the middle-third Cantor set, now takes the following form

$$
A \mathbf{C}=\bigcup_{s \in \mathcal{S}}(\mathbf{C}+s)
$$

where $A \mathbf{C}:=\{A x \mid x \in \mathbf{C}\}$, and $\mathbf{C}+s:=\{x+s \mid x \in \mathbf{C}\}$, or equivalently

$$
\mathbf{C}=\bigcup_{s \in \mathcal{S}} \sigma_{s}(\mathbf{C})
$$

where $\sigma_{s}(\mathbf{C}):=\left\{\sigma_{s}(x) \mid x \in \mathbf{C}\right\}$. The conditions on the pair $(A, \mathcal{S})$ guarantees that the sets in the union on the right-hand side in (3.7) or in (3.8), are mutually non-overlapping. This amounts to the so-called open-set-condition of Hutchinson [12]. The set $\mathcal{R}$ which is defined in Proposition 2.1 in the special case of the middle-third Cantor set is now instead

$$
\mathcal{R}=\bigcup_{n \geq 0} \bigcup_{k \in \mathbb{Z}^{d}} A^{-n}(\mathbf{C}+k)=\mathcal{R}=\bigcup_{n \in \mathbb{Z}} \bigcup_{k \in \mathbb{Z}^{d}} A^{-n}(\mathbf{C}+k)
$$

where $\mathbf{C}$ is the (unique) compact set determined by (3.8), of Hutchinson's theorem [12]. The properties of Proposition 2.1 carry over mutatis mutandis, for example, the argument from Section 2 shows that for every $k \in \mathbb{Z}^{d}$ and every $n \in \mathbb{Z}$,

$$
\mathcal{R}+A^{-n} k=\mathcal{R}, \quad \text { and } A^{n} \mathcal{R}=\mathcal{R} .
$$

The Hilbert space $H$ from Definition 2.6 is now $H:=L^{2}\left(\mathcal{R}, \mathcal{H}^{h}\right)$. The unitary operators $T$ and $U$ from $(2.8-2.9)$ are now

$$
\left(T_{k} f\right)(x):=f(x-k), \quad f \in H, x \in \mathcal{R}, k \in \mathbb{Z}^{d}
$$

and

$$
(U f)(x)=\frac{1}{\sqrt{\#(\mathcal{S})}} f\left(A^{-1} x\right), \quad f \in H, x \in \mathcal{R} .
$$

The commutation relation from proposition 2.7 in its general form is

$$
U T_{k} U^{-1}=T_{A k}, \quad k \in \mathbb{Z}^{d} .
$$

We now need the familiar duality between the two groups $\mathbb{Z}^{d}$, and $\mathbb{T}^{d}=$ $\mathbb{R}^{d} / \mathbb{Z}^{d}$, which identifies points $n \in \mathbb{Z}^{d}$ with monomials on $\mathbb{T}^{d}$ as follows,

$$
z^{n}=z_{1}^{n_{1}} z_{2}^{n_{2}} \cdots z_{d}^{n_{d}}=e^{i 2 \pi n_{1} \theta_{1}} e^{i 2 \pi n_{2} \theta_{2}} \cdots e^{i 2 \pi n_{d} \theta_{d}} .
$$

Note that (3.13) identifies the torus $\mathbb{T}^{d}$ with the $d$-cube

$$
\left\{\left(\theta_{1}, \ldots, \theta_{d}\right) \mid 0 \leq \theta_{i}<1, i=1, \ldots, d\right\} .
$$


Since $\mathbf{C}$ is naturally identified with a subset of $\mathbb{T}^{d}$, we may view the monomials $\left\{z^{n} \mid n \in \mathbb{Z}^{d}\right\}$ as functions on $\mathbf{C}$ by restriction. We say that the system $(A, \mathcal{S})$ is of orthogonal type if there is a subset $\mathcal{T}$ of $\mathbb{Z}^{d}$ such that the set of functions $\left\{z^{n} \mid n \in \mathcal{T}\right\}$ is an orthonormal basis (ONB) in the Hilbert space $L^{2}\left(\mathbf{C}, \mu_{(A, \mathcal{S})}\right)$. If there is no subset $\mathcal{T}$ with this ONB-property we say that $(A, \mathcal{S})$ is of non-orthogonal type. The authors of [16] showed that $(4,\{0,2\})$ is of orthogonal type, while $(3,\{0,2\})$ is not. So for the Cantor set $\mathbf{C}_{4}$ there is an ONB $\left\{z^{n} \mid n \in \mathcal{T}\right\}$ for a subset $\mathcal{T}$ of $\mathbb{Z}$; in fact we may take

$$
\mathcal{T}=\{0,1,4,5,16,17,20,21,24,25, \ldots\}=\left\{\sum_{0}^{\text {finite }} n_{i} 4^{i} \mid n_{i} \in\{0,1\}\right\}
$$

For the middle-third Cantor set $\mathbf{C}_{3}$ it can be checked that $\left\{z^{n} \mid n \in \mathbb{Z}\right\}$ contains no more than two elements which are orthogonal in $L^{2}\left(\mathbf{C}_{3}, \mu_{3}\right)$.

Theorem 3.1 Let $(A, \mathcal{S})$ be an affine IFS in $\mathbb{R}^{d}$, and suppose $\mathcal{S}$ has an extension to a set of $A$-residues in $\mathbb{Z}^{d}$. Let

$$
h=\frac{\log \#(\mathcal{S})}{\log |\operatorname{det} A|},
$$

and let $(\mathbf{C}, \mu)$ be as above; i.e., depending on $(A, \mathcal{S})$, and let $\mathcal{R}$ be defined from $\mathbf{C}$ in the usual way as in (3.9). Assume further that

$$
\mathbf{C} \cap(\mathbf{C}+k)=\emptyset, \quad\left(k \in \mathbb{Z}^{d} \backslash\{0\}\right) .
$$

Then the system $(A, \mathcal{S})$ is of orthogonal type if and only iff there is a subset $\mathcal{T}$ in $\mathbb{Z}^{d}$ such that

$$
\begin{array}{r}
\left\{(\#(\mathcal{S}))^{n / 2} e^{i 2 \pi A^{n} k \cdot x} \chi_{\mathbf{C}}\left(A^{n} x-\ell\right) \mid k \in \mathcal{T},\left(n=0 \text { and } \ell \in \mathbb{Z}^{d}\right)\right. \text { or } \\
(n \geq 1 \text { and } \ell \neq \equiv \bmod A \text { for all } s \in \mathcal{S})\}
\end{array}
$$

is an orthonormal basis in the Hilbert space $L^{2}\left(\mathcal{R}, \mathcal{H}^{h}\right)$.

Remark 3.2 The significance of the assumption (3.15) is illustrated in [4]. Also note that (3.15) is automatically satisfied if $\mathbf{C}=\mathbf{C}(A, \mathcal{S})$ is contained in a $\mathbb{Z}^{d}$-tile. This is the case for the example $(A, \mathcal{S})=(4,\{0,2\})$, but there are examples in $d=2$ where it is not.

Proof. A simple check shows that

$$
\begin{aligned}
\mathcal{R}=\bigcup\left\{A^{-n}(\mathbf{C}+l) \mid\right. & \left(n=0 \text { and } \ell \in \mathbb{Z}^{d}\right) \\
& \text { or }(n \geq 1 \text { and } \ell \neq \equiv s \bmod A \text { for all } s \in \mathcal{S})\},
\end{aligned}
$$

and the union is disjoint. 
Suppose $(A, \mathcal{S})$ is of orthogonal type. We saw in Section 2 that the restriction of the Hausdorff measure $\mathcal{H}^{h}$ to $\mathbf{C}$ agrees with the Hutchinson measure $\mu=\mu_{(A, \mathcal{S})}$ on $\mathbf{C}=\mathbf{C}_{(A, \mathcal{S})}$. Hence density of $\left\{z^{n} \mid n \in \mathcal{T}\right\}$ in $L^{2}(\mathbf{C}, \mu)$ implies density of $\left\{e^{i 2 \pi k \cdot x} \chi_{\mathbf{C}}(x) \mid k \in \mathcal{T}\right\}$ in the subspace $L^{2}\left(\mathbf{C}, \mathcal{H}^{h}\right)$ of $L^{2}\left(\mathcal{R}, \mathcal{H}^{h}\right)$. Now the formula for $\mathcal{R}$ implies that the functions in (3.16) are dense in $L^{2}\left(\mathcal{R}, \mathcal{H}^{h}\right)$.

Suppose conversely that the family (3.16) is dense in $L^{2}\left(\mathcal{R}, \mathcal{H}^{h}\right)$. Then $\left\{z^{n} \mid n \in \mathcal{T}\right\}$ must be dense in $L^{2}(\mathbf{C}, \mu)$ since $\mathbf{C}$ is the support of Hutchinson's measure $\mu$, and since $\mu$ restricts $\mathcal{H}^{h}$.

Corollary 3.3 Let $\left(\mathbf{C}_{4}, \mu_{4}\right)$ be the Cantor construction in the unit interval $I \cong \mathbb{T}^{1}$ defined by the IFS $\sigma_{0}(x)=\frac{x}{4}, \sigma_{2}(x)=\frac{x+2}{4} ;$ i.e., by $(A, \mathcal{S})=$ $(4,\{0,2\})$, and let $\mathcal{R}$ be the subset of $\mathbb{R}$ defined in (3.9). Then the family of functions

$$
\begin{aligned}
\left\{2^{n / 2} e^{i 2 \pi 4^{n} k x} \chi_{\mathbf{C}}\left(4^{n} x-\ell\right) \mid\right. & k \in\{0,1,4,5,16,17, \ldots\} \\
\ell & \left.\in\left\{\begin{array}{cl}
\mathbb{Z} & \text { if } n=0 \\
\mathbb{Z} \backslash(4 \mathbb{Z}+\{0,2\}) & \text { if } n \geq 1 .
\end{array}\right\}\right\}
\end{aligned}
$$

forms an orthonormal basis in the Hilbert space $L^{2}\left(\mathcal{R}, \mathcal{H}^{\frac{1}{2}}\right)$.

Proof. This is a direct application of the theorem as the subset

$$
\mathcal{T}=\{0,1,4,5,16,17, \ldots\}
$$

from (3.14) and (3.17) satisfies the basis property for $\mathbf{C}_{4}, \mu_{4}$ by Theorem 3.4 in $[16]$.

The next result makes clear the notion of gap-filling wavelets in the context of iterated function systems (IFS). While it is stated just for a particular example, the idea carries over to general IFSs. Note that in the system (3.18) below of wavelet functions, the two $\psi_{2}$ and $\psi_{3}$ are gap-filling.

Corollary 3.4 Let $\mathbf{C}=\mathbf{C}_{4}$ be the Cantor set determined from the IFS, $\sigma_{0}(x)=\frac{x}{4}, \sigma_{2}(x)=\frac{x+2}{4}$, from the previous corollary. Then the three functions

$$
\begin{aligned}
& \psi_{1}(x):=\chi_{\mathbf{C}}(4 x)-\chi_{\mathbf{C}}(4 x-2) \\
& \psi_{2}(x):=\sqrt{2} \chi_{\mathbf{C}}(4 x-1) \\
& \psi_{3}(x):=\sqrt{2} \chi_{\mathbf{C}}(4 x-3)
\end{aligned}
$$

generate an orthonormal wavelet basis in the Hilbert space $L^{2}\left(\mathcal{R}, \mathcal{H}^{\frac{1}{2}}\right)$. Specifically, the family

$$
\left\{2^{\frac{k}{2}} \psi_{i}\left(4^{k} x-\ell\right) \mid i=1,2,3, k \in \mathbb{Z}, \ell \in \mathbb{Z}\right\}
$$

is an orthonormal basis in $L^{2}\left(\mathcal{R}, \mathcal{H}^{\frac{1}{2}}\right)$. 
Proof. We noted that our results in propositions 2.4 and 2.8 apply more generally to IFSs of affine type. So the result amounts to checking the general orthogonality relations for the functions $m_{0}, m_{1}, m_{2}, m_{3}$ on $\mathbb{T}$ which define wavelet filters for the system in (3.19). Note that from (3.19) the subband filters $\left\{m_{i}\right\}_{i=0}^{3}$ are as follows, $z \in \mathbb{T}$ :

$$
\begin{aligned}
& m_{0}(z)=\frac{1}{\sqrt{2}}\left(1+z^{2}\right) \\
& m_{1}(z)=\frac{1}{\sqrt{2}}\left(1-z^{2}\right) \\
& m_{2}(z)=z \\
& m_{3}(z)=z^{3} .
\end{aligned}
$$

Since the $4 \times 4$ matrix in the system

$$
\left(\begin{array}{l}
m_{0}(z) \\
m_{1}(z) \\
m_{2}(z) \\
m_{3}(z)
\end{array}\right)=\left(\begin{array}{cccc}
\frac{1}{\sqrt{2}} & 0 & \frac{1}{\sqrt{2}} & 0 \\
\frac{1}{\sqrt{2}} & 0 & -\frac{1}{\sqrt{2}} & 0 \\
0 & 1 & 0 & 0 \\
0 & 0 & 0 & 1
\end{array}\right)\left(\begin{array}{c}
1 \\
z \\
z^{2} \\
z^{3}
\end{array}\right)
$$

is clearly unitary, the result follows from a direct computation; see also the proof of theorem 6.2.

To verify that the Ruelle operator $R=R_{m_{0}}$ given by

$$
(R f)(z)=\frac{1}{4} \sum_{w^{4}=z}\left|m_{0}(w)\right|^{2} f(w)=\frac{1}{4} \sum_{w^{4}=z}\left(1+\frac{w^{2}+w^{-2}}{2}\right) f(w)
$$

satisfies the two conditions

(a) $\operatorname{dim}\{f \in C(\mathbb{T}) \mid R f=f\}=1$, and

(b) for all $\lambda \in \mathbb{C},|\lambda|=1$, and $\lambda \neq 1, \operatorname{dim}\{f \in C(\mathbb{T}) \mid R f=\lambda f\}=0$, we may again apply the theorem from [18] or the results of section 6 below.

For the more general affine IFSs the results above extend as follows. Consider the affine IFS $\left(\sigma_{i}\right)_{i=1}^{p}$ with

$$
\sigma_{i}(x)=\frac{1}{N}\left(x+a_{i}\right), \quad(x \in \mathbb{R}),
$$

where $N \geq 2$ is an integer and $\left(a_{i}\right)_{i=1}^{p}$ are distinct integers in $\{0, \ldots, N-1\}$. Then by [9], there is a unique compact subset $K$ of $\mathbb{R}$ which is the attractor of the IFS, i.e.,

$$
\mathbf{C}=\cup_{i=1}^{p} \sigma_{i}(\mathbf{C})
$$

Actually, one can give a more explicit description of this attractor, namely

$$
\mathbf{C}=\left\{\sum_{j \geq 1} d_{j} N^{-j} \mid d_{j} \in\left\{a_{1}, \ldots, a_{p}\right\}, j \geq 1\right\} .
$$


Since the digits $a_{i}$ are distinct and less then $N, K$ is contained in $[0,1]$, and the sets $\sigma_{i}(K)$ are almost disjoint (they have at most one point in common, those of the form $k / N$ for some $k \in\{1, \ldots, N-1\}$.

The Hausdorff dimension of $K$ is $\log _{N} p$.

Now consider the set $\mathcal{R}=\left\{\sum_{j \geq-m} d_{j} N^{-j} \mid m \in \mathbb{Z}, d_{j} \in\left\{a_{1}, \ldots, a_{p}\right\}\right.$ for all but finitely many indices $\left.j\right\}$ $\mathcal{R}$ is invariant under integer translations

$$
\mathcal{R}+k=\mathcal{R}, \quad(k \in \mathbb{Z}),
$$

and it is invariant under dilation by $N$

$$
N \mathcal{R}=\mathcal{R} .
$$

Endow $\mathcal{R}$ with the Hausdorff measure $\mathcal{H}^{s}$ for $s=\log _{N} p$, and on $L^{2}\left(\mathcal{R}, \mathcal{H}^{s}\right)$, define the translation operator

$$
T f(x)=f(x-1), \quad\left(x \in \mathcal{R}, f \in L^{2}\left(\mathcal{R}, \mathcal{H}^{s}\right)\right),
$$

and the dilation operator

$$
U f(x)=\sqrt{\frac{1}{p}} f\left(\frac{x}{N}\right), \quad\left(x \in \mathcal{R}, f \in L^{2}\left(\mathcal{R}, \mathcal{H}^{s}\right)\right) .
$$

These are unitary operators satisfying the commutation relation

$$
U T U^{-1}=T^{N} \text {. }
$$

Let $\varphi:=\chi_{\mathbf{C}}$. The function $\varphi$ is an orthogonal scaling function for $L^{2}\left(\mathcal{R}, \mathcal{H}^{s}\right)$, with filter

$$
m_{0}(z)=\sqrt{\frac{1}{p}} \sum_{i=1}^{p} z^{a_{i}},
$$

so it satisfies the following conditions:

1. [Orthogonality]

$$
\left\langle T^{k} \varphi \mid \varphi\right\rangle=\delta_{k}, \quad(k \in \mathbb{Z}) .
$$

2. [Scaling equation]

$$
U \varphi=\sum_{i=1}^{p} \sqrt{\frac{1}{p}} T^{a_{i}} \varphi=m_{0}(T) .
$$

3. [Cyclicity]

$$
\overline{\operatorname{span}}\left\{U^{-n} T^{k} \varphi \mid n, k \in \mathbb{Z}\right\}=L^{2}\left(\mathcal{R}, \mathcal{H}^{s}\right)
$$


Next, we define the wavelets. For this, we need the "high-pass" filters $m_{1}, \ldots, m_{N-1}$ such that the matrix

$$
\frac{1}{\sqrt{N}}\left(m_{i}\left(\rho^{j} z\right)\right)_{i, j=0}^{N-1}
$$

is unitary for almost every $z$. $\left(\rho=e^{2 \pi i / N}\right)$.

First, we define the filters for the gap-filling wavelets $\psi_{1}, \ldots, \psi_{N-p}$. The set $G=\{0, \ldots, N-1\} \backslash\left\{a_{1}, \ldots, a_{p}\right\}$ has $N-p$ elements. We label the functions $z \mapsto z^{d}$ for $d \in G$, by $m_{1}, \ldots, m_{N-p}$.

The remaining $p-1$ filters are for the detail-filling wavelets. Let $\eta=$ $e^{2 \pi i / p}$. Define

$$
m_{N-p+k}(z)=\sqrt{\frac{1}{p}} \sum_{i=1}^{p} \eta^{k(i-1)} z^{a_{i}}, \quad(k \in\{1, \ldots, p-1\}) .
$$

We have to check that

$$
\frac{1}{N} \sum_{w^{N}=z} m_{i}(w) \bar{m}_{j}(w)=\delta_{i j}, \quad(z \in \mathbb{T}, i, j \in\{0, \ldots, N-1\}) .
$$

For this we use the following identity:

$$
\sum_{w^{N}=z} w^{k}=0, \quad(z \in \mathbb{T}, k \not \equiv 0 \quad \bmod N) .
$$

Therefore, if $f_{1}(z)=\sum_{i=0}^{N-1} \alpha_{i} z^{i}, f_{2}=\sum_{i=0}^{N-1} \beta_{i} z^{i}$, then

$$
\frac{1}{N} \sum_{w^{N}=z} f_{1}(w) \overline{f_{2}(w)}=\frac{1}{N} \sum_{i, j=0}^{N-1} \alpha_{i} \bar{\beta}_{j} \sum_{w^{N}=z} w^{i-j}=\sum_{i=0}^{N-1} \alpha_{i} \bar{\beta}_{j} .
$$

Applying these to the filters $m_{i},(i \in\{0, \ldots, N-1\})$, we obtain (3.21).

With these filters, we construct the wavelets in the usual way:

$$
\psi_{i}=U^{-1} m_{i}(T) \varphi, \quad(i \in\{1, \ldots, N-1\}),
$$

and

$$
\left\{U^{m} T^{n} \psi_{i} \mid m, n \in \mathbb{Z}, i \in\{1, \ldots, N-1\}\right\}
$$

is an orthonormal basis for $L^{2}\left(\mathcal{R}, \mathcal{H}^{s}\right)$.

Let $N \in \mathbb{Z}_{+}$be as above, and consider $\mathcal{S}=\left\{a_{1}, \ldots, a_{p}\right\} \subset\{0,1,2, \ldots, N-1\}$. A second subset $\mathcal{B}=\left\{b_{1}, \ldots, b_{p}\right\} \subset \mathbb{Z}$ is an $N$-dual if the $p \times p$ matrix

$$
M_{N}(\mathcal{S}, \mathcal{B})=\frac{1}{\sqrt{p}}\left(\exp \left(i \frac{2 \pi a_{j} b_{k}}{N}\right)\right)_{1 \leq j, k \leq p}
$$

is unitary. 
When $N$ and $\mathcal{S}$ are given as specified, it is not always true that there is a subset $\mathcal{B} \subset \mathbb{Z}$ for which $M_{N}(\mathcal{S}, \mathcal{B})$ is unitary. If for example $N=3$ and $\mathcal{S}=\{0,2\}$, then no $\mathcal{B}$ exists, while for $N=4$ and $\mathcal{S}=\{0,2\}$, we may take $\mathcal{B}=\{0,1\}$, and

$$
M_{4}(\mathcal{S}, \mathcal{B})=\frac{1}{\sqrt{2}}\left(\begin{array}{cc}
1 & 1 \\
1 & -1
\end{array}\right)
$$

is of course unitary.

Lemma 3.5 ([16]) Let $N$ and $\mathcal{S}$ be as specified above, and suppose

$$
\mathcal{B}=\left\{b_{1}, \ldots, b_{p}\right\} \subset \mathbb{Z}
$$

is an $N$-dual subset. Suppose $0 \in \mathcal{B}$, and set

$$
\Lambda=\Lambda_{N}(\mathcal{B}):=\left\{\sum_{i=0}^{\text {finite }} n_{i} N^{i} \mid n_{i} \in \mathcal{B}\right\} .
$$

Let $(\mathbf{C}, \mu)=\left(\mathbf{C}_{(N, \mathcal{S})}, \mu_{(N, \mathcal{S})}\right)$ be the Hutchinson pair. Then the set of functions $\left\{z^{n} \mid n \in \Lambda\right\}$ is orthogonal in $L^{2}(\mathbf{C}, \mu)$; i.e.,

$$
\int_{\mathbf{C}} z^{n-n^{\prime}} d \mu(z)=\delta_{n, n^{\prime}}, \quad n, n^{\prime} \in \Lambda
$$

where we identify $\mathbf{C}$ as a subset of $\mathbb{T}^{1}$ via

$$
\mathbf{C} \ni \theta \longrightarrow e^{i 2 \pi \theta} \in \mathbb{T}^{1} \text {. }
$$

Proof. Set $e(\theta)=e^{i 2 \pi \theta}$, and for $k \in \mathbb{R}$

$$
B(k):=\int_{\mathbf{C}} e(k \theta) d \mu(\theta) .
$$

Using (3.4), we get

$$
B(k)=\frac{1}{\sqrt{p}} m_{0}\left(\frac{k}{N}\right) B\left(\frac{k}{N}\right),
$$

where $m_{0}$ is defined in (3.20).

If $n, n^{\prime} \in \Lambda$, and $n \neq n^{\prime}$, we get the representation

$$
n^{\prime}-n=b^{\prime}-b+m N^{\ell}, \quad b, b^{\prime} \in \mathcal{B}, m, \ell \in \mathbb{Z}, \ell \geq 1 .
$$

As a result, the inner product in $L^{2}(\mathbf{C}, \mu)$ is

$$
\left\langle z^{n} \mid z^{n^{\prime}}\right\rangle_{\mu}=B\left(n^{\prime}-n\right)=\frac{1}{\sqrt{p}} m_{0}\left(\frac{b^{\prime}-b}{N}\right) B\left(\frac{n^{\prime}-n}{N}\right) .
$$

Since the matrix $M_{N}(\mathcal{S}, \mathcal{B})$ is unitary,

$$
m_{0}\left(\frac{b^{\prime}-b}{N}\right)=0
$$

when $b^{\prime} \neq b$ in $\mathcal{B}$, and the result follows. 
Even if the matrix $M_{N}(\mathcal{S}, \mathcal{B})$ is unitary, the orthogonal functions $\left\{z^{n} \mid\right.$ $n \in \Lambda$ \} might not form a basis for $L^{2}(\mathbf{C}, \mu)$. From [16], we know that it is an orthonormal basis (ONB) if and only if

$$
\sum_{n \in \Lambda}|B(\xi-n)|^{2}=1 \quad \text { a.e. } \xi \in \mathbb{R} .
$$

Introducing the function

$$
\Omega(\xi):=\frac{1}{p} \sum_{b \in \mathcal{B}}|B(\xi-n)|^{2},
$$

and the dual Ruelle operator

$$
\left(R_{\mathcal{B}} f\right)(\xi):=\frac{1}{p} \sum_{b \in \mathcal{B}}\left|m_{0}\left(\frac{\xi-b}{N}\right)\right|^{2} f\left(\frac{\xi-b}{N}\right),
$$

we easily verify that $\Omega$ and the constant function $\hat{1}$ both solve the eigenvalue problem $R_{\mathcal{B}}(f)=f$, both functions $\Omega$ and $\hat{1}$ are continuous on $\mathbb{R}$, even analytic.

Theorem 3.6 If the space

$$
\left\{f \in \operatorname{Lip}(\mathbb{R}) \mid f \geq 0, f(0)=1, \quad R_{\mathcal{B}}(f)=f\right\}
$$

is one-dimensional, then $\Lambda\left(=\Lambda_{N}(\mathcal{B})\right)$ induces an ONB; i.e., $\left\{z^{n} \mid n \in \Lambda\right\}$ is an $O N B$ in $L^{2}(\mathbf{C}, \mu)$.

Proof. The result follows from the discussion and the added observation that $\Omega(0)=1$. This normalization holds since $0 \in \mathcal{B}$ was assumed, and so $\left\langle e_{0} \mid e_{n}\right\rangle_{\mu}=0$ for all $n \in \Lambda \backslash\{0\}$.

Definition 3.7 A $\mathcal{B}$-cycle is a finite set $\left\{z_{1}, z_{2}, \ldots, z_{k+1}\right\} \subset \mathbb{T}$, with a pairing of points in $\mathcal{B}$, say $b_{1}, b_{2}, \ldots, b_{k+1} \in \mathcal{B}$, such that

$$
z_{i}=\sigma_{-b_{i}}\left(z_{i+1}\right), \quad z_{k+1}=z_{1},
$$

and $\left|m_{0}\left(z_{i}\right)\right|^{2}=p$.

Equivalently, a $\mathcal{B}$-cycle may be given by $\left\{\xi_{1}, \ldots, \xi_{k+1}\right\} \subset \mathbb{R}$ satisfying

$$
\begin{aligned}
\xi_{i+1} & \equiv b_{i}+N \xi_{i} \bmod N \mathbb{Z} \\
\left(N^{k}-1\right) \xi_{1} & \equiv b_{k}+N b_{k-1}+\cdots+N^{k-1} b_{1} \bmod N^{k} \mathbb{Z}
\end{aligned}
$$


Theorem 3.8 Let $N \in \mathbb{Z}_{+}, N \geq 2$ be given. Let $\mathcal{S} \subset\{0,1, \ldots, N-1\}$,

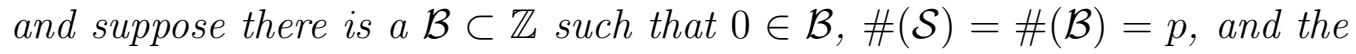
matrix

$$
M_{N}(\mathcal{S}, \mathcal{B})=\frac{1}{\sqrt{p}}\left(\exp \left(i \frac{2 \pi a b}{N}\right)\right)
$$

is unitary. Then $\left\{z^{n} \mid n \in \Lambda_{N}(\mathcal{B})\right\}$ is an $O N B$ for $L^{2}(\mathbf{C}, \mu)$ where $\Lambda_{N}(\mathcal{B})$ is defined in (3.23) if the only $\mathcal{B}$-cycles are the singleton $\{1\} \subset \mathbb{T}$.

Proof. By Theorem 3.6, we need only verify that the absence of $\mathcal{B}$-cycles of order $\geq 2$ implies that the Perron-Frobenius eigenspace (3.30) is onedimensional. But this follows from [6, Theorem 5.5.4]. In fact, the argument from Chapter 5 in [6] shows that the absence of $\mathcal{B}$-cycles of order $\geq 2$ implies that the $\mathcal{B}$-Ruelle operator $R_{\mathcal{B}}$ with $\sigma_{-b}(\xi):=\frac{\xi-b}{N}$,

$$
\left(R_{\mathcal{B}} f\right)(\xi)=\frac{1}{p} \sum_{b \in \mathcal{B}}\left(\left|m_{0}\left(\sigma_{-b}(\xi)\right)\right|^{2} f\left(\sigma_{-b}(\xi)\right)\right)
$$

satisfies the two Perron-Frobenius properties:

(i) the only bounded continuous solutions $f$ to $R_{\mathcal{B}}(f)=f$ are the multiples of $\hat{1}$, and

(ii) for all $\lambda \in \mathbb{T} \backslash\{1\}$, the eigenvalue problem $R_{\mathcal{B}}(f)=\lambda f$ has no non-zero bounded continuous solutions.

Example 3.9 (An Application) Let $N=4, \mathcal{S}=\{0,2\}$, and $\mathcal{B}=\{0,1\}$. Then

$$
\begin{aligned}
M_{4}(\mathcal{S}, \mathcal{B}) & =\frac{1}{\sqrt{2}}\left(\begin{array}{cc}
1 & 1 \\
1 & -1
\end{array}\right), \\
\Lambda_{4}(\mathcal{B}) & =\{0,1,4,5,16,17,20,21, \ldots\}, \text { and } \\
\left(R_{\mathcal{B}} f\right)(\xi) & =\cos ^{2}(2 \pi \xi) f\left(\frac{\xi}{4}\right)+\sin ^{2}(2 \pi \xi) f\left(\frac{\xi-1}{4}\right)
\end{aligned}
$$

and there is only on $\mathcal{B}$-cycle, the singleton $\{1\} \subset \mathbb{T}$. Recall from [12] that the Hutchinson construction of $(\mathbf{C}, \mu)$ identifies $\mathbf{C}$ as the Cantor set arising by the subdividing algorithm starting with the unit interval $I$ dividing into four equal subintervals and dropping the second and the fourth at each step in the algorithm. The measure $\mu$ is the restriction of $\mathcal{H}^{\frac{1}{2}}$ to $\mathbf{C}$, and it follows from the last theorem that $\left\{z^{n} \mid n \in \Lambda_{4}(\mathcal{B})\right\}$ is an ONB for $L^{2}(\mathbf{C}, \mu)$. The dual system $\left\{\sigma_{-b} \mid b \in \mathcal{B}\right\}$; i.e., $\sigma_{0}(\xi)=\frac{\xi}{4}, \sigma_{-1}(\xi)=\frac{\xi-1}{4}$, generates a Cantor subset $\mathbf{C}_{\mathcal{B}} \subset[-1,0]$ also of Hausdorff dimension $\frac{1}{2}$. Note that the fractional version of the Ruelle operator $R_{\mathcal{B}}$ does not map 1-periodic functions into themselves; in general

$$
\left(R_{\mathcal{B}} f\right)(\xi) \neq\left(R_{\mathcal{B}} f\right)(\xi+1)
$$


in fact

$$
\left(R_{\mathcal{B}} f\right)(\xi+1)=\cos ^{2}(2 \pi \xi) f\left(\frac{\xi+1}{4}\right)+\sin ^{2}(2 \pi \xi) f\left(\frac{\xi}{4}\right)
$$

so

$$
R_{\mathcal{B}} f(\xi)=R_{\mathcal{B}} f(\xi+1)
$$

holds only if

$$
\cos ^{4}(2 \pi \xi) f\left(\frac{\xi+1}{4}\right)=\sin ^{4}(2 \pi \xi) f\left(\frac{\xi-1}{4}\right) .
$$

The following tables of similar examples is included hopefully offering the reader a glimpse of the variety of examples, all of orthogonal type. The tables also offers some insight into the duality between the two systems, one in the $x$-variable and the other in the Fourier dual variable $\xi$

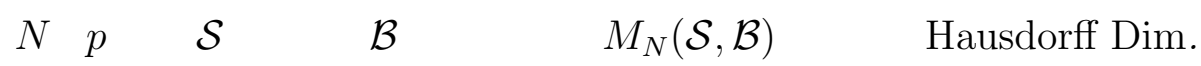

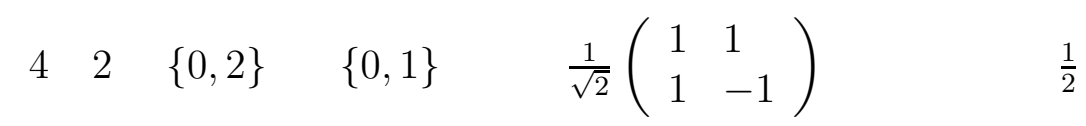

$$
\begin{aligned}
& \begin{array}{ll}
6 & 2
\end{array}\{, 3\} \quad\{0,1\} \quad \frac{1}{\sqrt{2}}\left(\begin{array}{ll}
1 & 1 \\
1 & -1
\end{array}\right) \quad \log _{6}(2)
\end{aligned}
$$

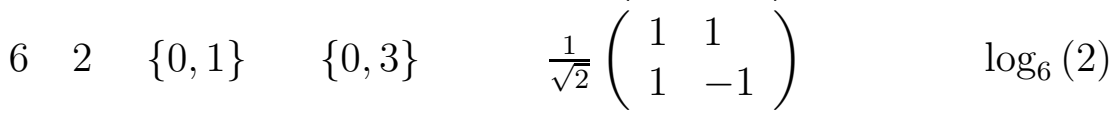

$$
\begin{aligned}
& \begin{array}{llllll}
6 & 3 & \{0,2,4\} & \{0,1,2\} & \frac{1}{\sqrt{3}}\left(\begin{array}{ccc}
1 & 1 & 1 \\
1 & \zeta_{3} & \zeta_{3}^{2} \\
1 & \zeta_{3}^{2} & \zeta_{3}
\end{array}\right) & \log _{6}(3) \\
& \text { where } \zeta_{3}=\exp \left(i \frac{2 \pi}{3}\right)
\end{array} \\
& N p \quad \Lambda_{N}(\mathcal{B}) \\
& 42 \quad\{0,1,4,5,16,17,20,21, \ldots\} \\
& 62 \quad\{0,1,6,7,36,37,42,43, \ldots\} \\
& 62\left\{\begin{array}{l}
6,3,6,9,36,39,42,45, \ldots\} \\
6
\end{array}\right. \\
& 63\{0,1,2,6,7,8,36,37,38,42,43,44, \ldots\} \\
& N p \quad\left(R_{B} f\right)(\xi) \\
& 42 \quad \cos ^{2}(2 \pi \xi) f\left(\frac{\xi}{4}\right)+\sin ^{2}(2 \pi \xi) f\left(\frac{\xi-1}{4}\right) \\
& 62 \cos ^{2}\left(\frac{\pi \xi}{2}\right) f\left(\frac{\xi}{6}\right)+\sin ^{2}\left(\frac{\pi \xi}{2}\right) f\left(\frac{\xi-1}{6}\right) \\
& 62 \cos ^{2}\left(\frac{\pi \xi}{6}\right) f\left(\frac{\xi}{6}\right)+\sin ^{2}\left(\frac{\pi \xi}{6}\right) f\left(\frac{\xi-3}{6}\right) \\
& 63 W\left(\frac{\xi}{6}\right) f\left(\frac{\xi}{6}\right)+W\left(\frac{\xi-1}{6}\right) f\left(\frac{\xi-1}{6}\right)+W\left(\frac{\xi-2}{6}\right) f\left(\frac{\xi-2}{6}\right) \\
& \text { where } W(\xi):=\frac{2 \cos ^{2}(2 \pi \xi)-\sin ^{2}(3 \pi \xi)}{3} \text {. }
\end{aligned}
$$




\section{A Generalized Zak-Transform}

The notion of filter is imported into math from signal processing. It has now been well adapted to wavelet analysis: In the familiar dyadic case the two wavelet functions $\varphi$ (the father function) and $\psi$ (the mother function) are in the Hilbert space $L^{2}(\mathbb{R})$. In the $N$-adic case, the wavelet functions are $\varphi, \psi_{1}, \ldots, \psi_{N-1}$, and there are known conditions for when these functions are in $L^{2}(\mathbb{R})$. The starting point is the scaling identity (1.6) satisfied by $\varphi$. Introducing the wavelet filter $m_{0}(z)=\sum_{k} a_{k} z^{k}$ as a function on $\mathbb{T}=\mathbb{R} / 2 \pi \mathbb{Z}$, and the transfer operator $R_{m_{0}}$ in (1.10), we note that necessary conditions for $\varphi$ to be in $L^{2}(\mathbb{R})$ are $\left|m_{0}(1)\right|=\sqrt{N}$, the low-pass condition, and $R_{m_{0}}(\hat{1})=\hat{1}$, where $\hat{1}$ is the constant function 1 . Let $\delta_{1}$ denote the Dirac mass at $z=1$. It follows that $\delta_{1} R_{m_{0}}=\delta_{1}$. But what if, for some $m_{0}, \delta_{1}$ does not satisfy this, so called low-pass condition; but rather there is some other probability measure $\nu$ on $\mathbb{T}$ which is $R_{m_{0}}$-invariant; i.e., satisfies $\nu R_{m_{0}}=\nu$, and which is singular with full support. A main point in our paper is that this alternative introduces fractal analysis into the wavelet construction.

Traditionally, the Zak-transform [7] is a standard tool of analysis in $L^{2}(\mathbb{R})$, and in this section it is extended to the abstract case of Hilbert space. If $\left\{T_{k}: k \in \mathbb{Z}\right\}$ denotes translation $\left(T_{k} f\right)(x)=f(x-k), x \in \mathbb{R}$, $k \in \mathbb{Z}, f \in L^{2}(\mathbb{R})$, we set

$$
(Z f)(z, x)=\sum_{k \in \mathbb{Z}} z^{k} T_{k} f(x), \quad z \in \mathbb{T}, x \in I,
$$

and we check that $Z$ defines a unitary isomorphism of $L^{2}(\mathbb{R})$ onto $L^{2}(\mathbb{T} \times I)$ where $\mathbb{T}$ is the torus, and $I$ the unit-interval $I=[0,1)$. The measure on $\mathbb{T}$ is Haar measure, denoted $\mu$; i.e.,

$$
\int_{\mathbb{T}} \cdots d \mu(z)=\frac{1}{2 \pi} \int_{0}^{2 \pi} \cdots d \theta, \quad \text { where } z=e^{i \theta} .
$$

Let $\mathcal{H}$ be a Hilbert space, $U$ a unitary operator in $\mathcal{H}$, and $T: \mathbb{Z} \rightarrow \mathcal{U}(\mathcal{H})$ a unitary representation. Let $N \geq 2$, and suppose that

$$
U T_{k} U^{-1}=T_{N k}, \quad k \in \mathbb{Z} .
$$

In general, the inner product in a Hilbert space $\mathcal{H}$ will be written $\langle\cdot \mid \cdot\rangle$. If $f \in \mathcal{H}$, then the form $g \rightarrow\langle f \mid g\rangle$ is taken linear on $\mathcal{H}$; and $\langle f \mid f\rangle=\|f\|^{2}$.

For $f_{i} \in \mathcal{H}, i=1,2$, we introduce the following function

$$
p\left(f_{1}, f_{2}\right)(z)=\sum_{k \in \mathbb{Z}} z^{k}\left\langle T_{k} f_{1} \mid f_{2}\right\rangle
$$

defined formally for $z \in \mathbb{T}$. 
Let $m_{0} \in L^{\infty}(\mathbb{T})$ be given, and suppose that

$$
\frac{1}{N} \sum_{w \in \mathbb{T}, w^{N}=z}\left|m_{0}(w)\right|^{2}=1, \quad \text { a.e. } \quad z \in \mathbb{T} .
$$

Note that the sum in (4.3) is finite, since for each $z \in \mathbb{T}$, the equation $w^{N}=z$ has precisely $N$ solutions. In fact, the cyclic group $\mathbb{Z}_{N}$ acts transitively on this set of solutions $\{w\}$.

(We will work with the torus $\mathbb{T}$ in anyone of its three familiar incarnations: (i) $\{z \in \mathbb{C}|| z \mid=1\}$, (ii) the quotient group $\mathbb{R} / 2 \pi \mathbb{Z}$, or (iii) the period interval $[0,2 \pi)$ via the identification $z=e^{i \theta}$. With this identification, we get the familiar description of the set $\left\{w \in \mathbb{T} \mid w^{N}=z\left(=e^{i \theta}\right)\right\}$ in the summation on the left-hand side of (4.3) as the $N$ distinct frequency bands $\left\{\frac{\theta+2 \pi k}{N} \mid k=0,1, \ldots, N-1\right\}$. The sub-interval $\left[0, \frac{2 \pi}{N}\right)$ represents the low frequency band.)

The operator $m_{0}(T)$ is defined from the spectral theorem in the usual way: If the spectral measure of $T$ is denoted $E_{T}$, then $E_{T}$ is a projection valued measure on $\mathbb{T}$, and we have the following three identities:

$$
\begin{aligned}
\|f\|^{2}=\int_{\mathbb{T}}\left\|E_{T}(d z) f\right\|^{2}, & f \in \mathcal{H}, \\
T_{k}=\int_{\mathbb{T}} z^{k} E_{T}(d z), & k \in Z,
\end{aligned}
$$

and by functional calculus,

$$
m_{0}(T)=\int_{\mathbb{T}} m_{0}(z) E_{T}(d z)
$$

If

$$
m_{0}(z)=\sum_{k \in \mathbb{Z}} a_{k} z^{k}
$$

is the Fourier series of $m_{0}$, it follows that $m_{0}(T)=\sum_{k \in \mathbb{Z}} a_{k} T_{k}$ is then well defined.

The following operator $R=R_{m_{0}}$, called the Ruelle operator, (see [15]) is acting on functions $h$ or $\mathbb{T}$ as follows,

$$
(R h)(z)=\frac{1}{N} \sum_{w \in \mathbb{T}, w^{N}=z}\left|m_{0}(w)\right|^{2} h(w), \quad z \in \mathbb{T} .
$$

Let $\hat{1}$ denote the constant function 1 on $\mathbb{T}$. Then condition (4.3) amounts to the eigenvalue equation

$$
R(\hat{1})=\hat{1}
$$


On the Hilbert space $\mathcal{H}$, we introduce the operator

$$
M:=U^{-1} m_{0}(T) .
$$

It is called the cascade approximation operator. In the special case when $T_{k} f(x)=f(x-k),(U f)(x)=N^{-\frac{1}{2}} f\left(\frac{x}{N}\right)$, and $\mathcal{H}=L^{2}(\mathbb{R})$, then

$$
(M f)(x)=\sqrt{N} \sum_{k} a_{k} f(N x-k)
$$

where $\left\{a_{k}: k \in \mathbb{Z}\right\}$ is the sequence of Fourier coefficients of $m_{0}$, see (4.7). In this case $M$ is also called the wavelet subdivision operator. The following general lemma applies also to the case of wavelets in $L^{2}(\mathbb{R})$. The advantage of (4.10) over (4.11) is that (4.10) is defined for all systems $U, T$ satisfying (4.1) and applies in particular to our present fractal examples.

For measurable functions $\xi$ and $\eta$ on $\mathbb{T}$, formula (4.6) represents the usual functional calculus; i.e., $\xi(T)=\int_{\mathbb{T}} \xi(z) E_{T}(d z)$. Setting $\pi(\xi):=\xi(T)$, we get $\pi(\xi \eta)=\pi(\xi) \pi(\eta), \pi(\hat{1})=I=$ the identity operator, $\pi(\bar{\xi})=\pi(\xi)^{*}=$ the adjoint operator. These properties together state that $\pi\left(=\pi_{E_{T}}\right)$ defines a *-representation of $L^{\infty}(\mathbb{T})$ acting on the Hilbert space $\mathcal{H}_{T}$ of the translation operators $\left\{T_{k}: k \in \mathbb{Z}\right\}$.

Lemma 4.1 Let $\mathcal{H}, T, U$, and $m_{0}$ be as described above, and let the operators $M$ and $R$ be the corresponding operators; i.e., the cascade operator, and Ruelle operator, respectively. Then the identity

$$
R\left(p\left(f_{1}, f_{2}\right)\right)=p\left(M f_{1}, M f_{2}\right)
$$

holds for all $f_{1,} f_{2} \in \mathcal{H}$, where the two sides in (4.12) are viewed as functions on $\mathbb{T}$.

Proof. Let $\xi \in C(\mathbb{T})$. Then it follows from (4.2) and (4.7) that

$$
\int_{\mathbb{T}} \xi(z) p\left(f_{1}, f_{2}\right)(z) d \mu(z)=\left\langle f_{1} \mid \xi(T) f_{2}\right\rangle
$$

where $\mu$ is the Haar measure on $\mathbb{T},\langle\cdot \mid \cdot\rangle$ is the inner product of $\mathcal{H}$, and $\xi(T)=\int_{\mathbb{T}} \xi(z) d E_{T}(z)$. Using this, in combination with (4.10), we therefore get

$$
\begin{aligned}
p\left(M f_{1}, M f_{2}\right)(z) & =\sum_{k \in \mathbb{Z}} z^{k}\left\langle T_{k} U^{-1} m_{0}(T) f_{1} \mid U^{-1} m_{0}(T) f_{2}\right\rangle \\
& =\sum_{k \in \mathbb{Z}} z^{k}\left\langle T_{N k} m_{0}(T) f_{1} \mid m_{0}(T) f_{2}\right\rangle \\
& =\frac{1}{N} \sum_{w \in \mathbb{T}, w^{N}=z} p\left(f_{1},\left|m_{0}\right|^{2}(T) f_{2}\right)(w)
\end{aligned}
$$


and therefore

$$
\begin{aligned}
\int_{\mathbb{T}} \xi(z) p\left(M f_{1}, M f_{2}\right)(z) d \mu(z) & =\int_{\mathbb{T}} \xi\left(z^{N}\right) p\left(f_{1},\left|m_{0}\right|^{2}(T) f_{2}\right)(z) d \mu(z) \\
& =\int_{\mathbb{T}} \xi\left(z^{N}\right)\left|m_{0}(z)\right|^{2} p\left(f_{1}, f_{2}\right)(z) d \mu(z) \\
& =\int_{\mathbb{T}} \xi(z) R\left(p\left(f_{1}, f_{2}\right)\right)(z) d \mu(z) .
\end{aligned}
$$

Since this is valid for all $\xi \in C(\mathbb{T})$, a comparison of the two sides in the last formula, now yields the desired identity (4.13).

Remark 4.2 An immediate consequence of the lemma is that if some $\varphi \in \mathcal{H}$ satisfies $M \varphi=\varphi$, or equivalently

$$
U \varphi=\sum_{k \in \mathbb{Z}} a_{k} T_{k} \varphi
$$

then the corresponding function $h:=p(\varphi, \varphi)$ on $\mathbb{T}$ satisfies $R(h)=h$. Recall that (4.14) is the scaling equation. If further the functions $\left\{T_{k} \varphi: k \in \mathbb{Z}\right\}$ on the right hand side in (4.14) can be chosen orthogonal, then

$$
p(\varphi, \varphi)(z)=\langle\varphi \mid \varphi\rangle_{\mathcal{H}}=\|\varphi\|_{\mathcal{H}}^{2}
$$

is the constant function, and so we are back to the special normalization condition (4.3) above.

The function $p(\varphi, \varphi)$ is called the auto-correlation function since its Fourier coefficients

$$
\int_{\mathbb{T}} z^{-k} p(\varphi, \varphi)(z) d \mu(z)=\left\langle T_{k} \varphi \mid \varphi\right\rangle_{\mathcal{H}}
$$

are the auto-correlation numbers.

Lemma 4.3 Let $m_{0} \in C(\mathbb{T})$ be given, and suppose (4.3) holds. that

(a) Then there is a probability measure $\nu=\nu_{m_{0}}$ depending on $m_{0}$ such

$$
\int_{\mathbb{T}} \xi d \nu=\int_{\mathbb{T}} R(\xi) d \nu
$$

holds for all $\xi \in C(\mathbb{T})$. 
(b) If $m_{0}$ is further assumed to be in the Lipschitz space $\operatorname{Lip}(\mathbb{T})$, then the following limit exists

$$
\lim _{n \rightarrow \infty} \frac{1}{n} \sum_{k=0}^{n-1} \mu R^{k}=\nu
$$

where $\mu R^{n}(\xi):=\mu\left(R^{n} \xi\right)=\int_{\mathbb{T}} R^{n} \xi d \mu, \mu$ is the Haar measure, and the convergence in (4.18) is in the Hausdorff metric (details below). The measure $\nu$ satisfies (4.17). Moreover, when then operator $R$ from $C(\mathbb{T})$ to $C(\mathbb{T})$ has Perron-Frobenius spectrum (i.e., 1 is the only eigenvalue of absolute value 1 ) then the limit

$$
\lim _{n \rightarrow \infty} \mu R^{n}=\nu,
$$

exists and gives the unique invariant measure $\nu$.

Definition 4.4 A function $\xi$ on $\mathbb{T}$ is said to be in $\operatorname{Lip}(\mathbb{T})$ if

$$
D \xi:=\sup _{-\pi \leq s<t<\pi}\left|\frac{\xi\left(e^{i s}\right)-\xi\left(e^{i t}\right)}{s-t}\right|<\infty .
$$

The Lipschitz-norm is $\|\xi\|_{\text {Lip }}:=D \xi+\xi(1)$.

The Hausdorff distance between two real valued measures $\nu_{1}, \nu_{2}$ on $\mathbb{T}$ is defined as

$$
\begin{aligned}
& \operatorname{dist}_{\text {Haus }}\left(\nu_{1}, \nu_{2}\right)= \\
& =\sup \left\{\int_{\mathbb{T}} \xi d \nu_{1}-\int \xi d \nu_{2} \mid \xi \in \operatorname{Lip}(T), \xi \text { real valued and } D \xi \leq 1\right\} .
\end{aligned}
$$

Hence the conclusion is the lemma states that if $m_{0} \in \operatorname{Lip}$ satisfies (4.3), then

$$
\lim _{n \rightarrow \infty} \operatorname{dist}_{\text {Haus }}\left(\nu, \mu R^{n}\right)=0 .
$$

Note that both the Ruelle operator $R$ and the measure $\nu$ depend on the function $m_{0}$. Condition (4.3) states that the constant function $\hat{1}$ is a rightPerron Frobenius eigenvector, and (4.17) that $\nu$ is a left-Perron-Frobenius eigenvector.

Proof of Lemma 4.3. The lemma is essentially a special case of the PerronFrobenius-Ruelle theorem, see [1]. Also note that an immediate consequence of (4.17) is the invariance

$$
\int_{\mathbb{T}} \xi\left(z^{N}\right) d \nu(z)=\int_{\mathbb{T}} \xi(z) d \nu(z)
$$


A key step in the proof is the following estimate: Let $\|\xi\|:=\sup _{z \in \mathbb{T}}|\xi(z)|$; then the following estimate

$$
\left\|R^{n} \xi\right\|_{\text {Lip }} \leq \frac{1}{N^{n}}\|\xi\|_{\text {Lip }}+2 D\left(\left|m_{0}\right|^{2}\right) \cdot\|\xi\|
$$

hold for all $\xi \in \operatorname{Lip}(\mathbb{T})$ and all $n \in \mathbb{Z}_{+}$. We leave the details of the verification of (4.24) to the reader; see also [6], and [17].

For functions $m$ and $m^{\prime}$ on $\mathbb{T}$, define the form $\left\langle m, m^{\prime}\right\rangle$ as a function on $\mathbb{T}$ as follows

$$
\left\langle m, m^{\prime}\right\rangle(z):=\frac{1}{N} \sum_{\substack{w \in \mathbb{T} \\ w^{N}=z}} \overline{m(w)} m^{\prime}(w) .
$$

Lemma 4.5 Let $N \geq 2$, and let $(\mathcal{H}, U, T)$ be a system which satisfies the commutation relation (4.1). Let $m$ and $m^{\prime}$ be functions on $\mathbb{T}$ which both satisfy the normalization condition (4.3). Let $M=M_{m}$ be the cascade approximation operator, $M=U^{-1} m(T)$, and suppose the scaling identity $M \varphi=\varphi$ has a non-zero solution in $\mathcal{H}$ such that the vectors $T_{k} \varphi, k \in \mathbb{Z}$, are mutually orthogonal. Let $M^{\prime}=M_{m^{\prime}}=U^{-1} m^{\prime}(T)$.

Then

$$
p\left(\varphi, M^{\prime} \varphi\right)(z)=\|\varphi\|_{\mathcal{H}}^{2} \cdot\left\langle m, m^{\prime}\right\rangle(z), \quad z \in \mathbb{T} .
$$

Proof. Using $M \varphi=\varphi$, and $p(\varphi, \varphi)(z)=\|\varphi\|_{\mathcal{H}}^{2}$, we get

$$
\begin{aligned}
p\left(\varphi, M^{\prime} \varphi\right)(z) & =p\left(M \varphi, M^{\prime} \varphi\right) \\
& =\frac{1}{N} \sum_{w^{N}=z} \overline{m(w)} m^{\prime}(w) p(\varphi, \varphi)(w) \\
& =\|\varphi\|_{\mathcal{H}}^{2} \cdot \frac{1}{N} \sum_{w^{N}=z} \overline{m(w)} m^{\prime}(w)=\|\varphi\|_{\mathcal{H}}^{2} \cdot\left\langle m, m^{\prime}\right\rangle(z)
\end{aligned}
$$

which is the desired identity (4.26) in the conclusion of the lemma.

Corollary 4.6 With the assumptions in Lemma 4.5, set

$$
m^{(n)}(z):=m(z) m\left(z^{N}\right) \ldots m\left(z^{N^{n-1}}\right)
$$

and

$$
m^{\prime(n)}(z):=m^{\prime}(z) m^{\prime}\left(z^{N}\right) \ldots m^{\prime}\left(z^{N^{n-1}}\right)
$$

Then

$$
p\left(\varphi, M^{\prime n} \varphi\right)(z)=\|\varphi\|_{\mathcal{H}}^{2} \cdot \frac{1}{N^{n}} \sum_{w^{N^{n}}=z} \overline{m^{(n)}(w)} m^{\prime(n)}(w)
$$

Proof. A direct iteration of the argument of Lemma 4.5 immediately yields the desired identity (4.28). 
Example 4.7 Let $N=3$, and let the functions $m$ and $m^{\prime}$ be given by $m(z)=\frac{1+z^{2}}{\sqrt{2}}$ and $m^{\prime}(z)=z^{3} m(z)$. Let

$$
\mathcal{H}=L^{2}\left(\mathcal{R},(d x)^{s}\right), s=\log _{3}(2),
$$

and $\varphi=\chi_{\mathbf{C}}$; i.e., $\varphi \in \mathcal{H}$ is the indicator function of the middle-third Cantor set, $\mathbf{C} \subset I$. Let $T f(x)=f(x-1)$, and $(U f)(x)=\frac{1}{\sqrt{2}} f\left(\frac{x}{3}\right)$. Then the conditions in Lemma 4.5 and Corollary 4.6 are satisfied for this system. Specifically $\|\varphi\|_{\mathcal{H}}=1, M \varphi=\varphi$ where $M=U^{-1} m(T)$; i.e.,

$$
\varphi(x)=\varphi(3 x)+\varphi(3 x-2),
$$

or

$$
3 \mathbf{C}=\mathbf{C} \cup(\mathbf{C}+2) .
$$

Also notice that

$$
M^{\prime}=U^{-1} m^{\prime}(T)=T M
$$

It follows that

$$
p\left(\varphi, M^{\prime n} \varphi\right)(z)=z^{1+3+3^{2}+\cdots+3^{n-1}}=z^{\frac{3^{n}-1}{2}}
$$

and therefore

$$
\left\langle\varphi, M^{\prime n} \varphi\right\rangle=\int_{\mathbb{T}} p\left(\varphi, M^{\prime n} \varphi\right)(z) d \mu(z)=0
$$

for all $n \in \mathbb{Z}_{+}$. In contrast to the standard cascade approximation for $L^{2}(\mathbb{R})$ with Lebesgue measure, we see that the cascade iteration on the Cantor function $\chi_{\mathbf{C}}$; i.e.,

$$
\chi_{\mathbf{C}}, M^{\prime} \chi_{\mathbf{C}}, M^{\prime 2} \chi_{\mathbf{C}}, \ldots
$$

does not converge in the Hilbert space $\mathcal{H}_{s}=L^{2}\left(\mathcal{R},(d x)^{s}\right)$. In fact the vectors in the sequence (4.33) are mutually orthogonal.

While the measure $\nu \in M(T)$ from Lemma 4.5 is generally not absolutely continuous with respect to the Haar measure $\mu$ on $\mathbb{T}$, the next result shows that it is the limit of the measures $\left|m^{(n)}(z)\right|^{2} d \mu(z)$ as $n \rightarrow \infty$ if the function $m$ is given to satisfy (4.3) and if the sequence $m^{(n)}$ is defined by (4.27).

Proposition 4.8 Let $m \in L^{\infty}(\mathbb{T})$ be given. Suppose (4.3) holds, and let $\nu$ be the Perron-Frobenius measure of Lemma 4.5; i.e., the measure $\nu=\nu_{m}$ arising as a limit (4.19). Then

$$
\lim _{n \rightarrow \infty} \int_{\mathbb{T}} \xi(z)\left|m^{(n)}(z)\right|^{2} d \mu(z)=\int_{\mathbb{T}} \xi(z) d \nu(z)
$$

holds for all $\xi \in C(\mathbb{T})$. 
Proof. Calculating the integrals on the left-hand side in (4.34), we get

$$
\int_{\mathbb{T}} \xi\left|m^{(n)}\right|^{2} d \mu=\int_{\mathbb{T}} \xi R^{*^{n}}(\hat{1}) d \mu=\int_{\mathbb{T}} R^{n}(\xi) d \mu \underset{n \rightarrow \infty}{\longrightarrow} \int_{\mathbb{T}} \xi d \nu
$$

where (4.18) was used in the last step. This is the desired conclusion (4.34) of the proposition.

Corollary 4.9 If $m(z)=\frac{1+z^{2}}{\sqrt{2}}$ is the function from Example 4.7, then the substitution $z=e^{i t}$ yields the following limit formula for the corresponding Perron-Frobenius measure $\nu$, written in multiplicative notation:

$$
\lim _{n \rightarrow \infty} \frac{1}{2 \pi} \prod_{k=1}^{n}\left(1+\cos \left(2 \cdot 3^{k} t\right)\right)=d \nu(t)
$$

Proof. The expression on the left-hand side in (4.35) is called a Rieszproduct, and it belongs to a wider family of examples; see for example [17] and [26]. The limit measure $\nu$ is known to be singular. It follows, for example from [26]. A computation shows that the Fourier coefficients $\hat{\nu}(n):=$ $\int_{\mathbb{T}} z^{n} d \nu(z)$, are real valued, satisfy $\hat{\nu}(0)=1, \hat{\nu}(1)=0, \hat{\nu}(-n)=\hat{\nu}(n)$, $\hat{\nu}(3 n)=\hat{\nu}(n), \hat{\nu}(3 n-2)=\frac{1}{2} \hat{\nu}(n), \hat{\nu}(3 n+2)=\frac{1}{2} \hat{\nu}(n)$, for all $n \in \mathbb{Z}$. Hence

$$
\lim \frac{1}{2 n+1} \sum_{k=-n}^{n}|\hat{\nu}(k)|^{2}=0=\text { sum of atoms; }
$$

i .e., $\sum|\nu(\{z\})|^{2}=0$. The last conclusion is from Wiener's theorem, and implies that $\nu$ has no atoms; i.e., $\nu(\{z\})=0$ for all $z \in \mathbb{T}$.

To check (4.36) some computations are required. Let

$$
s_{n}=|\hat{\nu}(0)|^{2}+|\hat{\nu}(1)|^{2}+\cdots+|\hat{\nu}(n)|^{2}, \quad(n \in \mathbb{N}) .
$$

Then, using the recursive relations for $\hat{\nu}$ we have

$$
\begin{aligned}
s_{3^{n+1}} & =s_{3^{n}}+\sum_{\substack{k=3^{n}+1 \\
k \equiv 0}}^{3^{n+1} \bmod 3}|\hat{\nu}(k)|^{2}+\sum_{\substack{k=3^{n}+1 \\
k=2} \bmod 3}^{3^{n+1}}|\hat{\nu}(k)|^{2}+\sum_{\substack{k=3^{n}+1 \\
k \equiv-2 \\
\bmod 3}}^{3^{n+1}}|\hat{\nu}(k)|^{2} \\
& \leq s_{3^{n}}+s_{3^{n}}+\frac{1}{4} s_{3^{n}}+\frac{1}{4} s_{3^{n}}=\frac{5}{2} s_{3^{n}} .
\end{aligned}
$$

By induction

$$
s_{3^{n}} \leq\left(\frac{5}{2}\right)^{n} s_{0}
$$


Now take $k$ arbitrary then for some $n, k$ is inbetween $3^{n}$ and $3^{n+1}$ so

$$
\frac{s_{k}}{k} \leq \frac{s_{3^{n+1}}}{3^{n}} \leq \frac{\left(\frac{5}{2}\right)^{n+1} s_{0}}{3^{n}}=\left(\frac{5}{6}\right)^{n} \frac{5}{2} s_{0},
$$

which shows that $s_{k} / k$ converges to 0 and this proves (4.36).

In summary, the measure $\nu$ is singular and non-atomic. In the next section we show that $\nu$ has full support.

Moreover there are supporting sets for $\nu$ which have zero Haar measure as subsets of $\mathbb{T}$. Concrete constructions are given below.

It follows from the recursive relations for the numbers $\hat{\nu}(n)$ that $\hat{\nu}(2 k+$ $1)=0, k \in \mathbb{Z}$; i.e., that all the odd Fourier coefficients vanish.

Each integer $n \in \mathbb{Z}_{+}$has a representation of the following form:

$$
n=l_{0}+l_{1} \cdot 3+l_{2} \cdot 3^{2}+\cdots+l_{p} \cdot 3^{p}, \quad l_{i} \in\{0,-2,2\}, i<p, l_{p} \in\{1,2\} .
$$

The representation is not unique (because for example $1=3 \cdot 1-2$ ) but uniqueness is obtain if we impose that $l_{p-1} \neq-2$ when $l_{p}=1$. We define a counting function $\#(n)$ which records the occurrence of values -2 and 2 for the 'trigets' $l_{i}$. Hence, if $n \in \mathbb{Z}_{+}$is even, then

$$
\hat{\nu}(n)=2^{-\#(n)} .
$$

Now, introduce the following sequence of functions

$$
g_{k}(z):=z^{2 \cdot 3^{k}}-\frac{1}{2}, \quad z \in \mathbb{T} .
$$

with inner products as follows with respect to the measure $\nu$ :

$$
\left\langle g_{k} \mid g_{l}\right\rangle_{\nu}=\int_{\mathbb{T}} \overline{g_{k}(z)} g_{l}(z) d \nu(z)=\frac{3}{4} \delta_{k, l}
$$

It follows from this that the series

$$
g(z):=\sum_{k=0}^{\infty} \frac{1}{k+1} g_{k}(z), \quad z \in \mathbb{T},
$$

is convergent in $L^{2}(\mathbb{T}, \nu)$ with

$$
\|g\|_{\nu}^{2}=\sum_{k=0}^{\infty} \frac{1}{(k+1)^{2}}\left\|g_{k}\right\|_{\nu}^{2}=\frac{\pi^{2}}{8} .
$$


Using the Riesz-Fisher theorem, we get a Borel subset $A \subset \mathbb{T}, \nu(A)=1$; i.e., $\nu(\mathbb{T} \backslash A)=0$, and a subsequence $n_{1}<n_{2}<n_{3}<\ldots, n_{i} \rightarrow \infty$, such that the series

$$
\sum_{k=0}^{n_{i}} \frac{1}{k+1} g_{k}(z)
$$

is pointwise convergent, $i \rightarrow \infty$, for all $z \in A$. But note that

$$
\sum_{k} \frac{1}{k+1} g_{k}(z)=\sum_{k} \frac{1}{k+1} z^{2 \cdot 3^{k}}-\frac{1}{2} \sum_{k} \frac{1}{k+1} .
$$

Using now Carleson's theorem about Fourier series on $\mathbb{T}$ with respect to Haar measure $\mu$ (=Lebesgue measure), we conclude that there is a Borel subset $B \subset \mathbb{T}, \mu(B)=1$; i.e., $\mu(\mathbb{T} \backslash B)=0$, such that the series

$$
\sum_{k=0}^{n} \frac{1}{k+1} z^{2 \cdot 3^{k}}
$$

is pointwise convergent, $n \rightarrow \infty$, for all $z \in B$. But identity (4.43) implies that $A \cap B=\varnothing$, and so $\mu(A)=0$. The supporting set $A$ for the measure $\nu$ has Lebesgue measure zero; and moreover the two measures $\nu$ and $\mu$ (= Haar measure on $\mathbb{T}$ ) are mutually singular.

It can be shown, using a theorem of Nussbaum [18], that the Ruelle operator

$$
(R f)(z)=\frac{1}{3} \sum_{w^{3}=z}|m(w)|^{2} f(w)=\frac{1}{3} \sum_{w^{3}=z}\left(1+\frac{w^{2}+w^{-2}}{2}\right) f(w)
$$

has Perron-Frobenius spectrum on $C(\mathbb{T})$, specifically that

$$
E_{R}(1)=\{f \in C(\mathbb{T}) \mid R f=f\}=\mathbb{C} \hat{1},
$$

and if $\lambda \in \mathbb{C},|\lambda|=1$, and $\lambda \neq 1$, that

$$
E_{R}(\lambda)=\{f \in C(\mathbb{T}) \mid R f=\lambda f\}=0 .
$$

As a consequence we get that

$$
\left\|R^{n}(f)-\nu(f)\right\|_{\text {Lip }} \leq \frac{1}{3^{n}}\|f\|_{\text {Lip }}
$$

holds for all $f \in \operatorname{Lip}(\mathbb{T})$ where $\|\cdot\|_{\text {Lip }}$ denotes the Lipschitz norm on functions on $\mathbb{T}$. 


\section{The support of Perron-Frobenius measures}

In this section we consider the support of the measure $\nu$. Caution: By the support of $\nu$, we mean the support of $\nu$ when it is viewed as a distribution; i.e., the support of $\nu$ is the complement of the union of the open subsets in $\mathbb{T}$ where $\nu$ acts as the zero distribution, or the zero Radon measure. Even though the support of $\nu$ may be all of $\mathbb{T}$, there can still be Borel subsets $E \subset \mathbb{T}$ with $\nu(E)=1$, but $E$ having zero Lebesgue measure.

Theorem 5.1 Let $m_{0} \in \operatorname{Lip}(\mathbb{T})$. Suppose (4.3) holds and $m_{0}$ has finitely many zeros and let $\nu$ be the Perron-Frobenius measure of Lemma 4.3. Then exactly one of the following affirmations is true:

(i) The support of $\nu$ is $\mathbb{T}$.

(ii) $\nu$ is atomic and the support of $\nu$ is a union of cycles $C=\left\{z_{1}, \ldots, z_{p}\right\}$ with $z_{1}^{N}=z_{2}, \ldots, z_{p-1}^{N}=z_{p}, z_{p}^{N}=z_{1}$, and $\left|m_{0}\right|^{2}\left(z_{i}\right)=N$ for all $i \in\{1, \ldots, p\}$. (Such cycles are called $\left(m_{0}, N\right)$-cycles $)$.

Proof. To simplify the notation, we use

$$
W:=\left|m_{0}\right|^{2} .
$$

Note that proposition 4.8 gives us $\nu$ as an infinite product $\prod_{k=1}^{\infty} W\left(z^{N^{k}}\right) d \mu$. In the next lemma we analyze the measures given by the tails of this product.

Lemma 5.2 Fix $n \geq 0$. We then have:

(i) For all $f \in C(\mathbb{T})$ the following limit exists, and defines a measure on $\mathbb{T}: \nu_{n}(f):=\lim _{k \rightarrow \infty} \int_{\mathbb{T}} W\left(z^{N^{n}}\right) \cdots W\left(z^{N^{n+k}}\right) f(z) d \mu$

(ii) $\nu_{n}(f)=\nu\left(R_{1}^{n} f\right),\left(f \in C(\mathbb{T})\right.$ where $R_{1}$ is given as in (4.8) but with $m_{0}=1$.

(iii) $\int_{\mathbb{T}} f(z) W(z) \cdots W\left(z^{N^{n-1}}\right) d \nu_{n}=\int_{\mathbb{T}} f(z) d \nu, f \in C(\mathbb{T})$. (So $\nu$ is absolutely continuous with respect to $\nu_{n}$.)

iv) $\lim _{n \rightarrow \infty} \nu_{n}(f)=\mu(f), f \in C(\mathbb{T})$.

Proof of Lemma. We can use a change of variable to compute

$$
\begin{aligned}
\lim _{k \rightarrow \infty} \int_{\mathbb{T}} W\left(z^{N^{n}}\right) \cdots W\left(z^{N^{n+k}}\right) f(z) d \mu= \\
\quad=\lim _{k \rightarrow \infty} \int_{\mathbb{T}} W(z) \cdots W\left(z^{N^{k}}\right) R_{1}^{n} f(z) d \mu=\nu\left(R_{1}^{n} f\right) .
\end{aligned}
$$

This proves (i) and (ii). (iii) is immediate from proposition 4.8 . 
For (iv) note that for $f \in C(\mathbb{T})$ and $n \in \mathbb{N}$.

$$
R_{1}^{n} f(\theta)=\frac{1}{N^{n}} \sum_{k=0}^{N^{n}-1} f\left(\frac{\theta+2 k \pi}{N^{n}}\right)
$$

therefore $R_{1}^{n} f$ converges uniformly to $\int_{\mathbb{T}} f d \mu$.

Then, with (ii), $\lim _{n \rightarrow \infty} \nu_{n}(f)=\mu(f)$.

We continue now the proof of the theorem. We distinguish two cases:

Case I: All measures $\nu_{n}$ are absolutely continuous with respect to $\nu$. In this case we prove that the support of $\nu$ is $\mathbb{T}$. Assume the contrary. Then there is an open set $U$ with $\nu(U)=0$. This implies $\nu_{n}(U)=0$ for all $n$.

Take $f \in C(\mathbb{T})$ with support contained in $U$. Then $\nu_{n}(f)=0$. Take the limit and use lemma 5.2 (iv), it follows that $\mu(f)=0$. As $f$ is arbitrary, $\mu(U)=0$. But this implies $U=\varnothing$, so the support of $\nu$ is indeed $\mathbb{T}$.

Case II: There is an $n \in \mathbb{N}$ such that $\nu_{n}$ is not absolutely continuous with respect to $\nu$. This means that there is a Borel set $E$ with $\nu(E)=0$ and $\nu_{n}(E)>0$.

We prove that there is a zero of $W^{(n)}$, call it $z_{0}$, such that $\nu_{n}\left(\left\{z_{0}\right\}\right)>0$. Suppose not. Then, take $E^{\prime}=E \backslash \operatorname{zeros}\left(W^{(n)}\right), \nu\left(E^{\prime}\right)=0 \nu_{n}\left(E^{\prime}\right)>0$.

The measure $\nu$ is regular so there is a compact subset $K$ of $E^{\prime}$ such that $\nu_{n}(K)>0$. Of course $\nu(K)=0$. Since $K$ has no zeros of $W^{(n)}$ and this is continuous, $W^{(n)}$ is bounded away from 0 on $K$. Then, with lemma 5.2(iii)

$$
0=\int_{K} \frac{1}{W^{(n)}(z)} d \nu=\int_{K} W^{(n)}(z) \frac{1}{W^{(n)}(z)} d \nu_{n}=\nu_{n}(K)
$$

which is a contradiction.

Thus, there is a $z_{0} \in \operatorname{zeros}\left(W^{(n)}\right)$ with $\nu\left(\left\{z_{0}\right\}\right)>0$.

We know also that $\nu(f)=\nu(R f)$ for all $f \in C(\mathbb{T})$. By approximation (Lusin's theorem) the same equality is true for all bounded Borel functions. Then

$$
\begin{aligned}
0<\nu\left(\chi_{\left\{z_{0}\right\}}\right) & =\nu\left(R \chi_{\left\{z_{0}\right\}}\right)=\nu\left(\frac{1}{N} \sum_{w^{N}=z} W(w) \chi_{\left\{z_{0}\right\}}(w)\right) \\
& =\frac{W\left(z_{0}\right)}{N} \nu\left(\chi_{\left\{z_{0}^{N}\right\}}\right) .
\end{aligned}
$$

Therefore $W\left(z_{0}\right)>0$ and $\nu\left(\left\{z_{0}^{N}\right\}\right)>0$. By induction, $W\left(z_{0}^{N^{k}}\right)>0$ and $\nu\left(\left\{z_{0}^{N^{k}}\right\}\right)$ for all $k \in \mathbb{N}$.

Since (4.3) holds, $W(z) \leq N$ for all $z \in \mathbb{T}$; so from the previous computation we obtain

$$
\nu\left(\left\{z^{N}\right\}\right) \geq \nu(\{z\}) .
$$


Since $\nu$ is a finite measure, the orbit $\left\{z_{0}^{N^{k}} \mid k \in \mathbb{N}\right\}$ has to be finite so the points $z_{0}, z_{0}^{N}, \ldots, z_{0}^{N^{p}}$ will form a cycle, $z_{0}^{N^{p+1}}=z_{0}$. Also,

$$
\nu\left(\left\{z_{0}\right\}\right)=\nu\left(\left\{z_{0}^{N^{p+1}}\right\}\right) \geq \nu\left(\left\{z_{0}^{N^{p}}\right\}\right) \geq \cdots \geq \nu\left(\left\{z_{0}\right\}\right)
$$

hence all inequalities are in fact equalities and with (5.1), this shows that $W\left(z_{0}^{N^{k}}\right)=N$ for $k \in\{0, \ldots, p\}$.

We are now in the "classical" case and we can use corollary 2.18 in [8] (See also [6]) to conclude that $\nu$ must be atomic and supported on cycles as mentioned in the theorem.

Lemma 5.3 Let $m_{0}, m_{0}^{\prime}$ be Lipschitz, with finitely many zeros. Suppose

$$
R_{m_{0}}(\hat{1})=\hat{1}=R_{m_{0}^{\prime}}(\hat{1}),
$$

and suppose there are no $m_{0}$ or $m_{0}^{\prime}$-cycles. Assume in addition that $m_{0}$ and $m_{0}^{\prime}$ have the same Perron-Frobenius measure $\nu$, then $\left|m_{0}\right|=\left|m_{0}^{\prime}\right|$.

Proof. With $W:=\left|m_{0}\right|^{2}$, we have, from proposition 4.8 , for $f \in C(\mathbb{T})$ :

$$
\lim _{n \rightarrow \infty} \int_{\mathbb{T}} f(z) W^{(n)}(z) d \mu(z)=\int_{\mathbb{T}} f(z) d \nu(z) .
$$

Then

$$
\lim _{n \rightarrow \infty} \int_{\mathbb{T}} f(z) W^{(n)}\left(z^{N}\right) d \mu=\lim _{n \rightarrow \infty} \int_{\mathbb{T}} R_{1} f(z) W^{(n)}(z) d \mu=\int_{\mathbb{T}} R_{1} f(z) d \nu .
$$

Take $f \in C(\mathbb{T})$ such that $f$ is zero in a neighborhood of zeros $\left(m_{0}\right)$. Then $\frac{f}{\left|m_{0}\right|^{2}}$ is continuous. So

$$
\lim _{n \rightarrow \infty} \int_{\mathbb{T}} \frac{f(z)}{\left|m_{0}(z)\right|^{2}} W^{(n)}(z) d \mu(z)=\int_{\mathbb{T}} \frac{f(z)}{\left|m_{0}(z)\right|^{2}} d \nu(z) .
$$

On the other hand

$$
\begin{aligned}
\lim _{n \rightarrow \infty} \int_{\mathbb{T}} \frac{f(z)}{\left|m_{0}(z)\right|^{2}} W^{(n)}(z) d \mu(z) & =\lim _{n \rightarrow \infty} \int_{\mathbb{T}} \frac{f(z)}{\left|m_{0}(z)\right|^{2}}\left|m_{0}(z)\right|^{2} \cdots\left|m_{0}\left(z^{N^{n-1}}\right)\right|^{2} d \mu \\
& =\lim _{n \rightarrow \infty} \int_{\mathbb{T}} f(z) W^{(n-1)}\left(z^{N}\right) d \nu(z) \\
& =\int_{\mathbb{T}} R_{1} f(z) d \nu .
\end{aligned}
$$

Thus

$$
\int_{\mathbb{T}} \frac{f(z)}{\left|m_{0}(z)\right|^{2}} d \nu(z)=\int_{\mathbb{T}} R_{1} f(z) d \nu(z)
$$

for all $f \in C(\mathbb{T})$ which are zero in a neighborhood of zeros $\left(m_{0}\right)$. 
The same argument can be applied to $m_{0}^{\prime}$. But note that the right-hand side of (5.2) doesn't depend on $m_{0}$ or $m_{0}^{\prime}$ (because $\nu$ is the same).

Therefore

$$
\int_{\mathbb{T}} \frac{f(z)}{\left|m_{0}(z)\right|^{2}} d \nu(z)=\int_{\mathbb{T}} \frac{f(z)}{\left|m_{0}^{\prime}(z)\right|^{2}} d \nu(z)
$$

for all $f \in C(\mathbb{T})$ which are zero on a neighborhood of zeros $\left(m_{0}\right) \cup \operatorname{zeros}\left(m_{0}^{\prime}\right)$.

From (5.3) it follows that $\left|m_{0}\right|=\left|m_{0}^{\prime}\right|, \nu$-almost everywhere on

$$
C:=\mathbb{T} \backslash\left(\operatorname{zeros}\left(m_{0}\right) \cup \operatorname{zeros}\left(m_{0}^{\prime}\right)\right) .
$$

Since the support of $\nu$ is $\mathbb{T}$, this implies that $\left|m_{0}\right|=\left|m_{0}^{\prime}\right|$ on a set which is dense in $\mathbb{T}$, and since the zeros of $m_{0}$ and $m_{0}^{\prime}$ are finite in number, $\left|m_{0}\right|=\left|m_{0}^{\prime}\right|$ on a dense subset of $\mathbb{T}$. By continuity therefore

$$
\left|m_{0}\right|=\left|m_{0}^{\prime}\right| \text { on } \mathbb{T}
$$

If $m_{0}$ and $W:=\left|m_{0}\right|^{2}$ are not assumed continuous, there is still a variant of Theorem 5.1, but with a weaker conclusion. For functions $W$ on $\mathbb{T}$ we introduce the following axioms. The a.e. conditions are taken with respect to the Haar measure $\mu$ on $\mathbb{T}$ :

(i) $W \in L^{\infty}(\mathbb{T})$,

(ii) $W \geq 0$ a.e. on $\mathbb{T}$ with respect to $\mu$,

(iii) $\frac{1}{N} \sum_{w^{N}=z} W(w)=1$ a.e. on $\mathbb{T}$,

(iv) The limit

$$
d \nu_{W}:=\lim _{n \rightarrow \infty} \frac{1}{n} \sum_{k=1}^{n} W^{(k)} d \mu
$$

exists in $M_{1}(\mathbb{T})$. Here, as before,

$$
W^{(k)}(z):=W(z) W\left(z^{N}\right) \cdots W\left(z^{N^{k-1}}\right),
$$

and

$$
\left(R_{W} f\right)(z)=\frac{1}{N} \sum_{w^{N}=z} W(w) f(w), \quad z \in \mathbb{T}, \quad f \in C(\mathbb{T})
$$


Theorem 5.4 Let $N \in \mathbb{Z}_{+}$, and let a function $W$ be given on $\mathbb{T}$ satisfying (i)-(iv) above. Let $\nu_{W}, W^{(k)}$ and $R_{W}$ be given as in (5.4)-(5.6). Then (a) - (c) hold:

(a) $\nu_{W}\left(R_{W}(f)\right)=\nu_{W}(f), \quad f \in C(\mathbb{T})$.

(b) If $W_{0}$, and $W$ both satisfy $(i)-(i v)$, set

$$
d \nu_{0}:=\lim _{n \rightarrow \infty} \frac{1}{n} \sum_{k=1}^{n} W_{0}^{(k)} d \mu,
$$

and

$$
f \longrightarrow \nu_{0}\left(R_{W}\left(f W_{0}\right)\right)
$$

defines a measure on $\mathbb{T}$ which is absolutely continuous with respect to $\nu_{0}$ with Radon-Nikodym derivative $W$; i.e., we have

$$
\nu_{0}\left(R_{W}\left(f W_{0}\right)\right)=\nu_{0}(f W), \quad f \in C(\mathbb{T}) .
$$

(c) If $W_{0}$ and $W$ are as in (b), then the following are equivalent:

(c $\left.c_{1}\right) \nu_{0} R_{W}=\nu_{0}$ and

$\left(c_{2}\right)$ there is a Borel subset $E \subset \mathbb{T}$ such that $\nu_{0}(E)=1$ and

$$
W_{0}(z)=W(z)
$$

for all $z \in E$.

Proof. The structure of this proof is as that of Theorem 5.1, the essential step consists of the following two duality identities. Each one amounts to a basic property of the Haar measure $\mu$ on $\mathbb{T}$. For every $k \in \mathbb{Z}_{+}$and $f \in C(\mathbb{T})$, we have

$$
\int_{\mathbb{T}} R_{W}(f) W^{(k)} d \mu=\int_{\mathbb{T}} f W^{(k+1)} d \mu
$$

and

$$
\int_{\mathbb{T}} R_{W}\left(f W_{0}\right) W_{0}^{(k)} d \mu=\int_{\mathbb{T}} f W W_{0}^{(k+1)} d \mu .
$$

Using these, and (5.2) for the measure $\nu_{0}$, the desired conclusions follow as before. For (c), in particular, we note that $\left(c_{1}\right)$ yields the identity $W d \nu_{0}=$ $W_{0} d \nu_{0}$ for the two functions $W_{0}$ and $W$ on $\mathbb{T}$. Hence $W_{0}$ and $W$ must agree on a Borel subset in $\mathbb{T}$ of full $\nu_{0}$-measure, and conversely. 


\section{Transformation Rules}

If $m_{0}: \mathbb{T} \rightarrow \mathbb{C}$ is a Fourier polynomial; i.e., represented by a finite sum $m_{0}(z)=\sum_{k} a_{k} z^{k}$, and if

$$
\frac{1}{N} \sum_{w^{N}=z}\left|m_{0}(w)\right|^{2}=1, \quad z \in \mathbb{T},
$$

for some $N \in \mathbb{Z}_{+}, N \geq 2$ we showed in [5] that there are functions $m_{1}, \ldots, m_{N-1}$ on $\mathbb{T}$ such that

$$
\frac{1}{N} \sum_{w^{N}=z} \overline{m_{j}(w)} m_{k}(w)=\delta_{j, k}, \quad z \in \mathbb{T},
$$

or equivalently, the $N \times N$ matrix

$$
\frac{1}{\sqrt{N}}\left(m_{j}\left(e^{i \frac{k \cdot 2 \pi}{N}} z\right)\right)_{j, k=0}^{N-1}, \quad z \in \mathbb{T}
$$

is unitary; i.e., defines a function from $\mathbb{T}$ into the group $U_{N}(\mathbb{C})$ of all unitary $N \times N$ matrices. Moreover the functions $m_{1}, \ldots, m_{N-1}$ may be chosen to be Fourier polynomials of the same total degree as $m_{0}$. For the example in Section $2, N=3$, and $m_{0}(z)=\frac{1+z^{2}}{\sqrt{2}}$, condition (6.1) is satisfied, and the other two functions may be chosen as: $m_{1}(z)=z$, and $m_{2}(z)=\frac{1-z^{2}}{\sqrt{2}}$. There is a more general result, also from [5] which defines a transitive action of the group $G_{N}$ of all unitary matrix functions $\left(G_{N}\right.$ is often called the $N$ th order loop-group, and it is used in homotopy theory). An element in $G_{N}$ is a function $A: \mathbb{T} \rightarrow U_{N}(\mathbb{C})$. Let $F_{N}$ denote all the functions $m=\left(m_{j}\right)_{j=0}^{N-1}: \mathbb{T} \rightarrow \mathbb{C}^{N}$ which satisfy (6.2), or equivalently (6.3). Define the action of $A$ on $m$ as follows:

$$
m^{A}(z):=A\left(z^{N}\right) m(z), \quad z \in \mathbb{T}
$$

Lemma 6.1 The action of $G_{N}$ on $F_{N}$ is transitive and effective; specifically, for any two $m$ and $m^{\prime} \in F_{N}$, there is a unique $A \in G_{N}$ such that $m^{\prime}=m^{A}$; i.e., A transforms $m$ to $m^{\prime}$.

Proof. For functions $f$ and $g$ on $\mathbb{T}$,

$$
\langle f, g\rangle_{N}(z):=\frac{1}{N} \sum_{w^{N}=z} \overline{f(w)} g(w), \quad z \in \mathbb{T} .
$$

If $m, m^{\prime} \in F_{N}$ are given, set

$$
A_{j, k}(z):=\left\langle m_{k}, m_{j}\right\rangle_{N}(z), \quad z \in \mathbb{T} .
$$

Then an easy verification shows that $A=\left(A_{j, k}\right)_{j, k=0}^{N-1}$ defines an element in $G_{N}$ which transforms $m$ to $m^{\prime}$. Conversely, if $m \in F_{N}$ is given, and $m^{A}$ is defined by (6.4), then it follows that $m^{A} \in F_{N}$ if and only if $A \in G_{N}$. 
If a function $m_{0}: \mathbb{T} \rightarrow \mathbb{C}$ is given, and satisfies (6.1) for some $N$, then the Ruelle transfer operator

$$
(R f)(z):=\frac{1}{N} \sum_{w^{N}=z}\left|m_{0}(w)\right|^{2} f(w), \quad z \in \mathbb{T}
$$

satisfies $R \hat{1}=\hat{1}$ where $\hat{1}$ denotes the constant function 1 on $\mathbb{T}$.

By a probability measure on $\mathbb{T}$, we mean a (positive) Borel measure $\nu$ on $\mathbb{T}$ such that $\nu(\mathbb{T})=1$. The probability measure will be denoted $M_{1}(\mathbb{T})$.

Terminology for measures $\nu$ on $\mathbb{T}$ : If $f \in C(\mathbb{T})$, set

$$
\nu(f):=\int_{\mathbb{T}} f d \nu=\int_{\mathbb{T}} f(z) d \nu(z) .
$$

If $\tau: \mathbb{T} \rightarrow \mathbb{T}$ is a measurable transformation, set

$$
\nu^{\tau^{-1}}(E)=\nu\left(\tau^{-1}(E)\right)
$$

for Borel sets $E \subset \mathbb{T}$.

If $R: C(\mathbb{T}) \rightarrow C(\mathbb{T})$ is linear, set

$$
(\nu R)(f)=\nu(R f)=\int_{\mathbb{T}}(R f) d \nu
$$

If $m_{0}$ is given as in (2.13), we introduce

$$
\mathcal{L}\left(m_{0}\right):=\left\{\nu \in M_{1}(\mathbb{T}): \nu R_{m_{0}}=\nu\right\} .
$$

If $\nu \in \mathcal{L}\left(m_{0}\right)$ and $E \subset \mathbb{T}$ is a Borel subset, we recall that $E$ supports $\nu$ if $\nu(E)=1$. Note that from the examples in Section 4, it may be that the support of $\nu$ is all of $\mathbb{T}$ even though $\nu$ has supporting Borel sets $E$ with zero Haar measure; i.e., $\nu(E)=1$ and $\mu(E)=0$.

We now return to the case of the middle-third Cantor set C. Set $s:=$ $\log _{3}(2)$, and view $\chi_{\mathbf{C}}$ as an element in the Hilbert space $L^{2}\left(\mathbb{R},(d x)^{s}\right)$. We recall the usual unitary operators $(U f)(x):=\frac{1}{\sqrt{2}} f\left(\frac{x}{3}\right)$, and $\left(T_{k} f\right)(x)=f(x-$ $k), k \in \mathbb{Z}$, and the relation

$$
U T_{k} U^{-1}=T_{3 k}, \quad k \in \mathbb{Z} .
$$

If $m_{0}(z)=\sum_{k} a_{k} z^{k}, m_{0} \in L^{\infty}(\mathbb{T})$, is given, we define the cascade approximation operator $M=M_{m_{0}}$ as before

$$
M f(x)=U^{-1} m_{0}(T) f(x)=\sqrt{2} \sum_{k \in \mathbb{Z}} a_{k} f(3 x-k), \quad f \in L^{2}\left(\mathbb{R},(d x)^{s}\right) .
$$

The condition

$$
\frac{1}{3} \sum_{w^{3}=z}\left|m_{0}(w)\right|^{2}=1 \text {, a.e. } z \in \mathbb{T},
$$

will be a standing assumption on $m_{0}$. 
We then define the sequence $m_{0}^{(n)}(z):=m_{0}(z) m_{0}\left(z^{3}\right) \cdots m_{0}\left(z^{3^{n-1}}\right)$, and we say that $m_{0}$ has frequency localization if the limit of an associated sequence of measures,

$$
\lim _{n \rightarrow \infty}\left|m_{0}^{(n)}(z)\right|^{2} d \mu(z)
$$

exists; i.e., if there is a $\nu \in M_{1}(\mathbb{T})$ such that

$$
\lim _{n \rightarrow \infty} \int_{\mathbb{T}} f(z)\left|m_{0}^{(n)}(z)\right|^{2} d \mu(z)=\int_{\mathbb{T}} f(z) d \nu(z)
$$

holds for all $f \in C(\mathbb{T})$. Recall that if $m_{0} \in \operatorname{Lip}(\mathbb{T})$ is assumed, and $R_{m_{0}}$ has Perron-Frobenius spectrum, then it has frequency localization, and the limit measure $\nu$ satisfies

$$
\lim _{n \rightarrow \infty} \operatorname{dist}_{\text {Haus }}\left(d \nu,\left|m_{0}^{(n)}\right|^{2} d \mu\right)=0 .
$$

Theorem 6.2 (The Dichotomy Theorem) Let $m_{0} \in L^{\infty}(\mathbb{T})$ be given, and suppose (6.10) holds, and let $\nu$ be the corresponding limit measure. Assume further that, for $k \in \mathbb{Z}_{+}$,

$$
\lim _{n \rightarrow \infty} \int_{\mathbb{T}}\left|m_{0}^{(n)}(z)\right|^{2} A_{0,0}^{(k)}(z) d \mu(z)=\nu\left(A_{0,0}^{(k)}\right),
$$

where $A$ is the matrix function defined in (6.6), and

$A^{(k)}(z):=A(z) A\left(z^{3}\right) \ldots A\left(z^{3^{k}}\right)$. Let $M=M_{m_{0}}$ be the cascade approximation operator in $L^{2}\left(\mathbb{R},(d x)^{s}\right), s=\log _{3}(2)$. Then the limit

$$
\lim _{n \rightarrow \infty} M^{n} \chi_{\mathbf{C}} \quad \text { exists in } L^{2}\left(\mathbb{R},(d x)^{s}\right)
$$

if and only if there is a Borel subset $E \subset \mathbb{T}$ such that $\nu(E)=1$ (i.e., $E$ is a supporting set for $\nu$ ), and $m_{0}(z)=\frac{1+z^{2}}{\sqrt{2}}$, for all $z \in E$. In the special case where $A$ is further assumed continuous and $m_{0}$ has frequency localization, the condition (6.13) is automatically satisfied,

$$
M f(x)=\left(M_{\mathbf{C}} f\right)(x)=f(3 x)+f(3 x-2)
$$

and

$$
M_{\mathbf{C}} \chi_{\mathbf{C}}=\chi_{\mathbf{C}} .
$$

Proof. Suppose first that (6.14) holds; i.e., that the cascading limit exists in $L^{2}\left(\mathbb{R},(d x)^{s}\right)$. Then in particular

$$
\lim _{n \rightarrow \infty}\left\|M^{n} \chi_{\mathbf{C}}-M^{n+1} \chi_{\mathbf{C}}\right\|_{L^{2}\left((d x)^{s}\right)}=0 .
$$


We saw, using [5] that there is a measurable matrix function $A: \mathbb{T} \rightarrow U_{3}(\mathbb{C})$ such that $m_{0}$ is the first component in the product, matrix times vector,

$$
\left(\begin{array}{l}
m_{0}(z) \\
m_{1}(z) \\
m_{2}(z)
\end{array}\right)=A\left(z^{3}\right)\left(\begin{array}{c}
\frac{1+z^{2}}{\sqrt{2}} \\
z \\
\frac{1-z^{2}}{\sqrt{2}}
\end{array}\right), \quad z \in \mathbb{T} .
$$

If the functions $A_{j, k}$ denote the entries in the matrix $A$ on the right-hand side in (6.18), we saw that

$$
p\left(\chi_{\mathbf{C}}, M \chi_{\mathbf{C}}\right)(z)=A_{0,0}(z), \quad z \in \mathbb{T}
$$

and

$$
\left\langle M^{n} \chi_{\mathbf{C}}, M^{n+1} \chi_{\mathbf{C}}\right\rangle_{L^{2}\left((d x)^{s}\right)}=\int_{\mathbb{T}} R^{n}\left(p\left(\chi_{\mathbf{C}}, M \chi_{\mathbf{C}}\right)\right) d \mu=\int_{\mathbb{T}} R^{n}\left(A_{0,0}\right) d \mu
$$

where

$$
R^{n}\left(A_{0,0}\right)(z)=\frac{1}{3^{n}} \sum_{w^{3^{n}}=z}\left|m_{0}^{(n)}(w)\right|^{2} A_{0,0}(w), \quad z \in \mathbb{T} .
$$

After a change of variable in (6.13), we conclude from (6.20) that

$$
\lim _{n \rightarrow \infty}\left\langle M^{n} \chi_{\mathbf{C}}, M^{n+1} \chi_{\mathbf{C}}\right\rangle_{L^{2}\left((d x)^{s}\right)}=\int_{\mathbb{T}} A_{0,0}(z) d \nu(z)=: \nu\left(A_{0,0}\right)
$$

and therefore

$$
0=\lim _{n \rightarrow \infty}\left\|M^{n} \chi_{\mathbf{C}}-M^{n+1} \chi_{\mathbf{C}}\right\|_{L^{2}\left((d x)^{s}\right)}^{2}=2-2 \operatorname{Re} \nu\left(A_{0,0}\right) .
$$

From (6.18), we know that $\left|A_{0,0}\right| \leq 1$, pointwise for $z \in \mathbb{T}$. From this, we get that $\nu\left(A_{0,0}\right)=1$. Hence, there is a Borel subset $E \subset \mathbb{T}$, such that $\nu(E)=1$, and $A_{0,0}(z)=1$ for all $z \in E$. Since

$$
\left|A_{0,0}(z)\right|^{2}+\left|A_{0,1}(z)\right|^{2}+\left|A_{0,2}(z)\right|^{2}=1, \quad z \in \mathbb{T},
$$

we conclude that $A_{0,1}(z)=A_{0,2}(z)=0$ for $z \in E$. Using (6.18) again, we finally get $m_{0}(z)=\frac{1+z^{2}}{\sqrt{2}}$ for $z \in E$; i.e., the conclusion of the theorem holds. If $A_{0,0}$ is assumed continuous, then $A_{0,0}=1$ on $\mathbb{T}$ since the support of $\nu$ is all of $\mathbb{T}$. The conclusions (6.15)-(6.16) in the theorem then follow.

We now turn to the converse implication: If some supporting set $E \subset \mathbb{T}$ exists such that $A_{0,0}(z)=1$ for $z \in E$, then

$$
\nu\left(A_{0,0}\right)=\int_{\mathbb{T}} A_{0,0} d \nu=\int_{E} A_{0,0} d \nu=\int_{E} d \nu=\nu(E)=1 .
$$


To prove the convergence in $L^{2}\left((d x)^{s}\right)$ of the cascades in (6.14), we must consider

$$
\left\|M^{n} \chi_{\mathbf{C}}-M^{n+k} \chi_{\mathbf{C}}\right\|_{L^{2}\left((d x)^{s}\right)}^{2}=2-2 \operatorname{Re} \int_{\mathbb{T}} R^{n}\left(p\left(\chi_{\mathbf{C}}, M^{k} \chi_{\mathbf{C}}\right)\right) d \mu,
$$

we note as in the case $k=1$, that

$$
p\left(\chi_{\mathbf{C}}, M^{k} \chi_{\mathbf{C}}\right)(z)=A_{0,0}^{(k)}(z) .
$$

If $z \in E$, then $A_{0,0}(z)=1$, and $A_{0, j}(z)=A_{j, 0}(z)=0$ for $j=1,2$.

As a result, using (6.23), we get

$$
A_{0,0}^{(2)}(z)=\sum_{j=0}^{2} A_{0, j}(z) A_{j, 0}\left(z^{3}\right)=A_{0,0}(z) A_{0,0}\left(z^{3}\right)=A_{0,0}\left(z^{3}\right),
$$

and therefore

$$
\begin{aligned}
\int_{E} A_{0,0}^{(2)}(z) d \nu(z) & =\int_{E} A_{0,0}\left(z^{3}\right) d \nu(z)=\int_{\mathbb{T}} A_{0,0}\left(z^{3}\right) d \nu(z) \\
& =\int_{\mathbb{T}} A_{0,0}(z) d \nu(z)=\nu\left(A_{0,0}\right)=1 .
\end{aligned}
$$

Continuing by induction, we find a supporting set, which is also denoted $E$, such that

$$
A_{0,0}^{(k)}(z)=1
$$

for all $z \in E, k=1,2, \ldots$. Since

$$
p\left(\chi_{\mathbf{C}}, M^{k} \chi_{\mathbf{C}}\right)(z)=A_{0,0}^{(k)}(z), \quad z \in \mathbb{T},
$$

substitution into (6.24) yields

$$
\left\|M^{n} \chi_{\mathbf{C}}-M^{n+k} \chi_{\mathbf{C}}\right\|_{L^{2}\left((d x)^{s}\right)}^{2}=2-2 \operatorname{Re} \int_{\mathbb{T}} R^{n}\left(A_{0,0}^{(k)}\right) d \mu \underset{n \rightarrow \infty}{\rightarrow} 2-2 \operatorname{Re} \nu\left(A_{0,0}^{(k)}\right)=0 .
$$

This proves convergence of the cascades, and concludes the proof of the theorem.

\section{Low pass filters}

For functions on the real line $\mathbb{R}$, and for every $N \in \mathbb{Z}_{+}, N \geq 2$, the scaling identity takes the form

$$
\varphi(x)=D \sum_{k \in \mathbb{Z}} a_{k} \varphi(N x-k)
$$

where $D$ is a dimensional fixed constant. We take $D=\sqrt{N}$. 
The values $a_{k}$ are called masking coefficients, and

$$
m_{0}(z):=D^{-1} \sum_{k \in \mathbb{Z}} a_{k} z^{k}
$$

is the corresponding low-pass filter. The terminology is from graphics algorithms and signal processing, and the books [7] and [6] explain this connection in more detail. The function $m_{0}$ is viewed as a function on $\mathbb{T}=\mathbb{R} / 2 \pi \mathbb{Z}$, or alternately as a $2 \pi$-periodic function on $\mathbb{R}$, via $z:=e^{-i \theta}, \theta \in \mathbb{R}$. It turns out that the regularity properties of $m_{0}$ are significant for the spectral theoretic properties which hold for the operators associated with $m_{0}$, specifically the cascade subdivision operator, and the Ruelle transfer operator. The function spaces which serve as repository for the function $m_{0}$ are measurable functions on $\mathbb{T}$, for example $L^{p}(\mathbb{T}), 1 \leq p \leq \infty$, the continuous functions; i.e., $C(\mathbb{T})$, or the Lipschitz functions $\operatorname{Lip}(\mathbb{T})$.

We will consider low-pass filters $m_{0}$ with the following properties:

(1) $m_{0} \in \operatorname{Lip}(\mathbb{T})$;

(2) $m_{0}$ has a finite number of zeros;

(3) $R_{m_{0}}(1)=1$.

Proposition 7.1 Suppose $m_{0}$ satisfies (1), (2), (3) and

$$
\operatorname{dim}\left\{h \in C(\mathbb{T}) \mid R_{m_{0}}(h)=h\right\} \geq 2 .
$$

Then there exists an $\left(m_{0}, N\right)$-cycle (i.e., a set $\left\{z_{1}, \ldots z_{p}\right\}$ with $z_{i}^{N}=z_{i+1}$, $z_{p}^{N}=z_{1}$ and $\left|m_{0}\left(z_{i}\right)\right|^{2}=N$ for all $\left.i\right)$.

Proof. Let $h \in C(\mathbb{T})$ satisfy $R_{m_{0}}(h)=h$, and $h$ non-constant. Taking the real or imaginary part, we may assume that $h$ is real valued. Also, replacing $h$ by $\|h\|_{\infty}-h$, we may assume $h \geq 0$, and that $h$ has some zeros.

We prove that all the zeros of $h$ must be cyclic points.

Suppose not, and let $z_{0} \in \mathbb{T}$ be a zero of $h$ which is not on a cycle. Then, $w_{1}^{N^{\ell_{1}}}=z_{0}$ and $w_{2}^{N^{\ell_{2}}}=z_{0}$ with $\ell_{1} \neq \ell_{2}$ implies $w_{1} \neq w_{2}$. Otherwise we have for some $\ell_{1}<\ell_{2}, z_{0}^{N^{\ell_{2}-\ell_{1}}}=w_{1}^{N^{\ell_{2}}}=z_{0}$ so $z_{0}$ is a cyclic point.

We say that $w$ is at level $\ell$ if $w^{N^{\ell}}=z_{0}$. The previous remark shows that $\ell$ is uniquely determined by $w$.

Since $h\left(z_{0}\right)=0$, it follows that

$$
\frac{1}{N} \sum_{w^{N}=z_{0}}\left|m_{0}(w)\right|^{2} h(w)=0,
$$

so $\left|m_{0}(w)\right|^{2} h(w)=0$ for all $w$ with $w^{N}=z_{0}$. Not all such $w^{\prime}$ s can have $m_{0}(w)=0$ because $R_{m_{0}}(1)=1$. Thus there is a $z_{1}$ with $z_{1}^{N}=z_{0}$ and $h\left(z_{1}\right)=0$.

By induction, there is a $z_{n+1}$ with $z_{n+1}^{N}=z_{n}$ and $h\left(z_{n+1}\right)=0$. 
Now, $m_{0}$ has only finitely many zeros, so from some level on, there are no zeros of $m_{0}$; i.e., if $w^{N^{\ell}}=z_{0}$ with $\ell \geq \ell_{0}$ then $m_{0}(w) \neq 0$. But then look at those $w$ 's with $w^{N}=z_{\ell_{0}}$. Since $h\left(z_{\ell_{0}}\right)=0$ and $m_{0}(w) \neq 0$, it follows that $h(w)=0$. By induction, $h(w)=0$ for all $w$ with $w^{N^{n}}=z_{0}$ and $n$ large enough.

However, these $w$ 's form a dense set because for any interval $(a, b) \subset \mathbb{T}$ there is an $m$ big enough such that $\tau^{m}(a, b)=\mathbb{T} \ni z_{\ell_{0}}\left(\right.$ where $\tau(z)=z^{N}$ ) so there is a $w \in(a, b)$ with $w^{N^{m}}=z_{0}$. Since $h$ is continuous, it follows that $h=0$, a contradiction.

Thus, all zeros of $h$ are cyclic points. In particular $z_{0}, z_{1}, \ldots z_{n}, \ldots$ are cyclic.

Since $z_{0}$ and $z_{1}$ are cyclic and $z_{1}^{N}=z_{0}$ (hence they are on the same cycle), if $w \neq z_{1}, w^{N}=z_{0}$ then $w$ is not cyclic so $h(w) \neq 0$, and therefore $m_{0}(w)=0$. But this implies (from $R_{m_{0}}(1)=1$ ) that $\left|m_{0}\left(z_{1}\right)\right|^{2}=N$. We can do the same for all terms of the cycle generated by $z_{1}$ and the conclusion of the proposition follows.

Remark 7.2 It is known generally that the dimension of the eigenspace in (7.3) depends on the metric properties of orbits in $\mathbb{T}$ under $z \rightarrow z^{N}$ where $N \geq 2$ is fixed. These orbits are called cycles. The solutions $h$ to

$$
R_{m_{0}}(h)=h
$$

are called $\left|m_{0}\right|^{2}$-harmonic functions and are important in the general theory of branching processes. Their significance for the present discussion is noted in [15]. There we prove that each solution $h \in L^{1}(\mathbb{T}), h \geq 0, h \neq 0$, to equation (7.4); i.e., a non-negative harmonic function, is naturally associated with a system $(H, U, T, \varphi)$ where $H$ is a Hilbert space, $U$ and $T$ are unitary operators in $H$ satisfying $U T U^{-1}=T^{N}$ (i.e., equation (4.1)), and the vector $\varphi \in H, \varphi \neq 0$, satisfies the general scaling identity

$$
U \varphi=m_{0}(T) \varphi, \quad \text { (i.e., the abstract form of (1.4) or (4.14).) }
$$

See also [3].

$T$ generates a representation of $L^{\infty}(\mathbb{T})$ by

$$
\pi(f)=f(T), \quad\left(f \in L^{\infty}(\mathbb{T})\right) .
$$

This representation satisfies the commutation relation

$$
U \pi(f) U^{-1}=\pi\left(f\left(z^{N}\right)\right), \quad\left(f \in L^{\infty}(\mathbb{T})\right) .
$$

Iterating the scaling identity one has

$$
U^{n} \varphi=\pi\left(m_{0}^{(n)}\right) \varphi, \quad(n \geq 0) .
$$

Moreover, the system $(H, U, \pi, \varphi)$ is determined from $h$ in (7.4) up unitary equivalence and it is called the wavelet representation associated to $\left(m_{0}, h\right)$. 
Returning to the form $p(\cdot, \cdot)$ in $(4.2)$, we note that $h$ is related to the new data by the two formulas,

$$
\begin{aligned}
h & =p(\varphi, \varphi) \quad \text { and } \\
\int_{\mathbb{T}} \xi\left(z^{N}\right) p(U \varphi, U \varphi)(z) d \mu(z) & =\int_{\mathbb{T}} \xi(z) h(z) d \mu(z), \quad \text { for all } \xi \in C(\mathbb{T}) .
\end{aligned}
$$

The paper [25] treats a general form of the problem (7.4), and these authors state that the number of $\left(m_{0}, N\right)$-cycles on $\mathbb{T}$ equals the dimension of the eigenspace in (7.3); i.e., the space of continuous $R_{m_{0}}$-harmonic functions. Recall the points $\left\{z_{i}\right\}$ on an $\left(m_{0}, N\right)$-cycle satisfy $\left|m_{0}\left(z_{i}\right)\right|=\sqrt{N}, z_{i+1}=z_{i}^{N}$, and $z_{k}=z_{0}$, if $k$ is the length of the cycle. The following example shows that this result from [25] is in need of a slight correction: Take for example $m_{0}(z)=\frac{1+z^{2}}{\sqrt{2}}, N=3$, where there are no $\left(m_{0}, 3\right)$-cycles. What is true is that, if the dimension in (7.3) is $>1$,or if there are eigenvalues $\lambda \in \mathbb{T} \backslash\{1\}$, then there must be $\left(m_{0}, N\right)$-cycles, and the arguments in [25] work: All the invariant measures are supported on cycles. But if the dimension in (7.3) is 1 , then there may, or may not, be $\left(m_{0}, N\right)$-cycles. If not, then the invariant measures have support equal to $\mathbb{T}$. If $\{1\}$ is an $\left(m_{0}, N\right)$-cycle, then Dirac's $\delta_{1}$ is an invariant measure.

We stress this distinction because it is central to explaining our dichotomy; i.e., explaining when a non-zero solution $\varphi$ exists to the scaling equation (4.14). If the given filter $m_{0}$ has an $\left(m_{0}, N\right)$-cycle of length 1 , then there is a scaling function $\varphi$ in $L^{2}(\mathbb{R})$, and we are in the classical (nonfractal) case. If $m_{0}$ and $N$ are fixed, but there is no $\left(m_{0}, N\right)$-cycle on $\mathbb{T}$, then $\varphi$ is instead in one of the $\mathcal{H}^{s}$-Hilbert spaces, $0<s<1$, with $s$ depending on the chosen particular iterated function system (IFS).

Proposition 7.3 Let $m_{0}$ be a filter that satisfies (1), (2), (3). If there exists $a \lambda \neq 1$ with $|\lambda|=1$ and $h \in C(\mathbb{T}), h \neq 0$, such that $R_{m_{0}}(h)=\lambda h$, then there exists an $\left(m_{0}, N\right)$-cycle.

Proof. We know that the peripheral eigenvalue spectrum of $R_{m_{0}}$ is a finite union of cyclic subgroups of $\mathbb{T}$ (see section 4.5 in [6]). Hence $\lambda^{n}=1$ for some $n$.

Then $R_{m_{0}}^{n}(h)=h$ so $R_{m_{0}^{(n)}}(h)=h$ and $h$ is not a constant. But $m_{0}^{(n)}$ is Lipschitz, it has finitely many zeros and $R_{m_{0}^{(n)}}(\hat{1})=\hat{1}$ (the scale for $m_{0}^{(n)}$ is $\left.N^{n}\right)$. Using proposition 7.1, it follows that there is an $\left(m_{0}^{(n)}, N^{n}\right)$ cycle; i.e., there exist points $z_{1}, \ldots, z_{p}$ on $\mathbb{T}$ with $z_{i}^{N^{n}}=z_{i+1}, z_{p}^{N^{n}}=z_{1}$, and $\left|m_{0}^{(n)}\left(z_{i}\right)\right|^{2}=N^{n}$. 
But $\left|m_{0}\right|^{2} \leq N$ (since $\left.R_{m_{0}}(1)=1\right)$ and this implies that

$$
\left|m_{0}\left(z_{i}\right)\right|^{2}=\left|m_{0}\left(z_{i}^{N}\right)\right|^{2}=\ldots=\left|m_{0}\left(z_{i}^{N^{n-1}}\right)\right|^{2}=N
$$

and therefore $z_{i}$ will generate an $\left(m_{0}, N\right)$-cycle.

Theorem 7.4 Suppose $m_{0}$ satisfies (1), (2), (3). Then exactly one of the following affirmations is true:

(i) There exists an $\left(m_{0}, N\right)$-cycle. In this case, the invariant measures $\nu$ with

$$
\nu\left(R_{m_{0}}(f)\right)=\nu(f),(f \in C(\mathbb{T}))
$$

are atomic supported on the $\left(m_{0}, N\right)$-cycles. The spectrum of $R_{m_{0}}$ is computed in [6] and [8]. The wavelet representation associated to $\left(m_{0}, 1\right)$ is a direct sum of cyclic amplifications of $L^{2}(\mathbb{R})$ (see [3]).

(ii) There are no $\left(m_{0}, N\right)$-cycles. In this case there are no eigenvalues for $R_{m_{0}} \mid C(\mathbb{T})$ with $|\lambda|=1$ other then $\lambda=1 ; 1$ is a simple eigenvalue. There exists a unique probability measure $\nu$ on $\mathbb{T}$ which is invariant for $R_{m_{0}}$ (i.e., $\nu\left(R_{m_{0}}(f)\right)=\nu(f)$ for $\left.f \in C(\mathbb{T})\right)$.

$$
\lim _{n \rightarrow \infty} R_{m_{0}}^{n}(f)=\nu(f) \text { uniformly } f \in C(\mathbb{T}) .
$$

Proof. When there are no $\left(m_{0}, N\right)$-cycles, proposition 7.1 and 7.3 show that there are no peripheral eigenvalues other than 1 and 1 is a simple eigenvalue. The statements about $\nu$ follow from theorem 3.4.4 and proposition 4.4.4 in [6] and their proofs.

Remark 7.5 When $m_{0}$ satisfies (1), (2), (3) and 1 is a simple eigenvalue for $\left.R_{m_{0}}\right|_{C(\mathbb{T})}$ then the invariant measure $\nu$ is unique and (7.5) holds (see [6] and [8], [3]). In the case of wavelet filters in $L^{2}(\mathbb{R}), m_{0}$ satisfies the extra condition

$$
m_{0}(1)=\sqrt{N}
$$

so $\{1\}$ is an $\left(m_{0}, N\right)$-cycle. The measure $\nu$ is simply the Dirac measure $\delta_{1}$.

Lemma 7.6 Let $m_{0}$ be a filter that satisfies (1), (2), (3). Assume in addition that 1 is a simple eigenvalue for $\left.R_{m_{0}}\right|_{C(\mathbb{T})}$. Consider the wavelet representation $(H, U, \pi, \varphi)$ associated to $\left(m_{0}, 1\right)$ as in remark 7.2.

Then for all $\xi \in H$ with $\|\xi\|=1$ and all $f \in C(\mathbb{T})$,

$$
\lim _{n \rightarrow \infty}\left\langle\xi \mid U^{-n} \pi(f) U^{n} \xi\right\rangle=\nu(f)
$$


Proof. First take $\xi$ of the form $\xi=U^{-m} \pi(g) \varphi$ with $m \in \mathbb{Z}, g \in C(\mathbb{T})$. Then, for $n>m$ :

$$
\begin{aligned}
\left\langle U^{-m}\right. & \pi(g) \varphi\left|U^{-n} \pi(f) U^{n} U^{-m} \pi(g) \varphi\right\rangle= \\
& =\left\langle\pi\left(g\left(z^{N^{n-m}}\right) m_{0}^{(n-m)}(z)\right) \varphi \mid \pi\left(f(z) g\left(z^{N^{n-m}}\right) m_{0}^{(n-m)}(z)\right) \varphi\right\rangle \\
& =\int_{\mathbb{T}} f(z)\left|g\left(z^{N^{n-m}}\right)\right|^{2}\left|m_{0}^{(n-m)}(z)\right|^{2} d \mu \\
& =\int_{\mathbb{T}}|g(z)|^{2} R_{m_{0}}^{(n-m)} f(z) d \mu .
\end{aligned}
$$

Since $\|\xi\|=1$, it follows that $\|\pi(g) \varphi\|=1$ so $\int_{\mathbb{T}}|g(z)|^{2} d \mu=1$. Also, from (7.5), $\lim _{n \rightarrow \infty} R_{m_{0}}^{n-m}(f)(z)=\nu(f)$ uniformly.

Therefore

$$
\lim _{n \rightarrow \infty}\left\langle\xi \mid U^{-n} \pi(f) U^{n} \xi\right\rangle=\int_{\mathbb{T}}|g(z)|^{2} \nu(f) d \mu=\nu(f) .
$$

Now take $\xi \in H$ arbitrarily, $\|\xi\|=1$. We can approximate $\xi$ by a sequence $\left(\xi_{j}\right)_{j}$ of the form mentioned before, with $\left\|\xi_{j}\right\|=1$.

Fix $\epsilon>0$. Then there is a $j$ such that $\left\|\xi_{j}-\xi\right\|<\left(\frac{\epsilon}{3}\right)\|f\|_{\infty}$ and there is an $n_{\epsilon}$ such that, for $n \geq n_{\epsilon}$,

$$
\left|\left\langle\xi_{j} \mid U^{-n} \pi(f) U^{n} \xi_{j}\right\rangle-\nu(f)\right|<\frac{\epsilon}{3} .
$$

Then

$$
\begin{aligned}
\mid\left\langle\xi \mid U^{-n} \pi(f) U^{n} \xi\right\rangle- & \nu(f) \mid \leq \\
\leq \mid & \left\langle\xi \mid U^{-n} \pi(f) U^{n} \xi\right\rangle-\left\langle\xi \mid U^{-n} \pi(f) U^{n} \xi_{j}\right\rangle \mid \\
& \quad+\left|\left\langle\xi \mid U^{-n} \pi(f) U^{n} \xi_{j}\right\rangle-\left\langle\xi_{j} \mid U^{-n} \pi(f) U^{n} \xi_{j}\right\rangle\right| \\
& \quad+\left|\left\langle\xi_{j}\left|U^{-n} \pi(f) U^{n} \xi_{j}\right|\right\rangle-\nu(f)\right| \\
\leq & \|f\|_{\infty}\left\|\xi-\xi_{j}\right\|\|\xi\|+\|f\|_{\infty}\left\|\xi_{j}\right\|\left\|\xi-\xi_{j}\right\|+\frac{\epsilon}{3}<\epsilon
\end{aligned}
$$

Theorem 7.7 Let $m_{0}, m_{0}^{\prime}$ be two filters satisfying (1), (2), (3) and suppose that 1 is a simple eigenvalue for $R_{m_{0}}$ and $R_{m_{0}^{\prime}}$ on $C(\mathbb{T})$. Let $\nu$ and $\nu^{\prime}$ be the invariant probability measures for $R_{m_{0}}$ and $R_{m_{0}^{\prime}}$ respectively and let $(H, U, \pi, \varphi),\left(H^{\prime}, U^{\prime}, \pi^{\prime}, \varphi^{\prime}\right)$ be the wavelet representations associated to $\left(m_{0}, 1\right)$ and $\left(m_{0}^{\prime}, 1\right)$ respectively.

If $\nu \neq \nu^{\prime}$ then the two wavelet representations are disjoint. 
Proof. Suppose the representations are not disjoint, then there is a partial isometry $W$ from $H$ to $H^{\prime}$, where $H$ and $H^{\prime}$ are the respective Hilbert spaces, and $W \neq 0$. Take $\xi$ in the initial space of $W,\|\xi\|=1$; then $\|W \xi\|=1$. Using lemma 7.6 we have for all $f \in C(\mathbb{T})$

$$
\begin{aligned}
\nu(f) & =\lim _{n \rightarrow \infty}\left\langle\xi \mid U^{-n} \pi(f) U^{n} \xi\right\rangle=\lim _{n \rightarrow \infty}\left\langle W \xi \mid W U^{-n} \pi(f) U^{n} \xi\right\rangle \\
& =\lim _{n \rightarrow \infty}\left\langle W \xi \mid U^{\prime-n} \pi^{\prime}(f) U^{\prime n} W \xi\right\rangle=\nu^{\prime}(f) .
\end{aligned}
$$

Thus $\nu=\nu^{\prime}$.

Corollary 7.8 Let $m_{0}$ be a filter that satisfies (1), (2), (3), and suppose 1 is a simple eigenvalue for $R_{m_{0}}$ on $C(\mathbb{T})$. Let $\nu$ be the invariant measure for $R_{m_{0}}$ and let $(H, U, \pi, \varphi)$ be the wavelet representation associated to $\left(m_{0}, 1\right)$. Suppose $\varphi^{\prime} \in H$ is another orthogonal scaling function with filter $m_{0}^{\prime}$. Then 1 is a simple eigenvalue for $R_{m_{0}^{\prime}}$ on $C(\mathbb{T})$. If $m_{0}^{\prime}$ satisfies also (1), (2) then the invariant measure $\nu^{\prime}$ for $R_{m_{0}^{\prime}}$ is equal to the one for $R_{m_{0}}$, i.e., $\nu^{\prime}=\nu$.

Proof. Repeating the calculation given in the proof of lemma 7.6 we have

$$
\begin{aligned}
\nu(f) & =\lim _{n \rightarrow \infty}\left\langle U^{-m} \pi(g) \varphi^{\prime} \mid U^{-n} \pi(f) U^{n}\left(U^{-m} \pi(g) \varphi^{\prime}\right)\right\rangle \\
& =\lim _{n \rightarrow \infty} \int_{\mathbb{T}}|g(z)|^{2} R_{m_{0}^{\prime}}^{n-m}(f) d \mu
\end{aligned}
$$

For all $f, g \in C(\mathbb{T}), m \in \mathbb{Z}, \int_{\mathbb{T}}|g|^{2} d \mu=1$. Suppose $h \in C(\mathbb{T}), h$ non constant with $R_{m_{0}^{\prime}}(h)=h$. Then

$$
\nu(h)=\int_{\mathbb{T}}|g(z)|^{2} h(z) d \mu
$$

for all $g \in C(\mathbb{T})$ with $\int_{\mathbb{T}}|g(z)|^{2} d \mu=1$ then $h$ is constant.

The last assertion follows directly from theorem 7.7.

Corollary 7.9 Let $m_{0}$ be a filter that satisfies (1), (2), (3) and suppose there are no $\left(m_{0}, N\right)$-cycles. Then the wavelet representation associated to $\left(m_{0}, 1\right)$ is disjoint from the classical wavelet representation on $L^{2}(\mathbb{R})$.

Proof. Since $\delta_{1}$ is not invariant for $R_{m_{0}}$, everything follows from theorem 7.7.

Acknowledgment. Toward the end of our work on this paper we had some very helpful discussions with Professors Steen Pedersen, Yang Wang, Roger Nussbaum and Richard Gundy on spectral theory of the Ruelle operators associated with IFSs and on infinite-product formulas. We thank them for many helpful suggestions. Corrections and helpful suggestions from the two referees are much appreciated. 


\section{References}

[1] Baladi, V.: Positive transfer operators and decay of correlations. Advanced Series in Nonlinear Dynamics 16. World Scientific Publishing, River Edge, NJ, 2000.

[2] Berry, M.: Making light of mathematics. Bull. Amer. Math. Soc. (N.S.) 40 (2003), 229-237.

[3] Bildea, S., Dutkay, D. and Picioroaga, G.: MRA super-wavelets. New York J. Math. 11 (2005), 1-19.(electronic).

[4] Bratteli, O. and Jorgensen, P. E. T.: Iterated function systems and permutation representations of the Cuntz algebra. Mem. Amer. Math. Soc. 139 (1999), 89 pp.

[5] Bratteli, O. and Jorgensen, P.E. T.: Wavelet filters and infinitedimensional unitary groups. In Wavelet analysis and applications (Guangzhou, 1999), 35-65. AMS/IP Stud. Adv. Math. 25. Amer. Math. Soc., Providence, RI, 2002.

[6] Bratteli, O. and Jorgensen, P.E.T.: Wavelets Through a Looking Glass. The world of the spectrum, chapter 2. Applied and Numerical Harmonic Analysis. Birkhäuser, Boston, MA, 2002.

[7] Daubechies, I.: Ten Lectures on Wavelets. CBMS-NSF Regional Conference Series in Applied Mathematics, 61. Society for Industrial and Applied Mathematics (SIAM), Philadelphia, PA, 1992.

[8] Dutkay, D.: The Wavelet Galerkin Operator. J. Operator Theory 51 (2004), no. 1, 49-70.

[9] Falconer, K. J.: The Geometry of Fractal Sets. Cambridge Tracts in Mathematics 85. Cambridge University Press, Cambridge, 1986.

[10] Gibbons, M., Raj, A. and Strichartz, R. S.: The finite element method on the Sierpinski gasket. Constr. Approx. 17 (2001), no. 4, 561-588.

[11] Huang, N. N. and Strichartz, R. S.: Sampling theory for functions with fractal spectrum. Experiment. Math. 10 (2001), no. 4, 619-638.

[12] Hutchinson, J. E.: Fractals and self-similarity. Indiana Univ. Math. J. 30 (1981) 713-747.

[13] Jonsson, A.: Wavelets on fractals and Besov spaces. J. Fourier Anal. Appl. 4 (1998), 329-340.

[14] Jonsson, A.: Haar wavelets of higher order on fractals and regularity of functions. J. Math. Anal. Appl. 290, (2004), 86-104.

[15] Jorgensen, P.E. T.: Ruelle Operators: functions which are harmonic with respect to a transfer operator. Mem. Amer. Math. Soc. 152 (2001), no. 720 .

[16] Jorgensen, P.E. T. And Pedersen, S.: Dense analytic subspaces in fractal $L^{2}$-spaces. J. Anal. Math. 75 (1998), 185-228. 
[17] Keane, M.: Strongly mixing g-measures. Invent. Math. 16 (1972), 309-324.

[18] Nussbaum, R. D.: Eigenvectors of order-preserving linear operators. J. London Math. Soc. (2) 58 (1998), 480-496.

[19] Sadun, L. and Williams, R. F.: Tiling spaces are Cantor set fiber bundles. Ergodic Theory Dynam. Systems 23 (2003), 307-316.

[20] Packer, J. A. And Rieffel, M. A.: Wavelet filter functions, the matrix completion problem, and projective modules over $C\left(\mathbb{T}^{n}\right)$. J. Fourier Anal. Appl. 9 (2003), 101-116.

[21] Strichartz, R. S.: Piecewise linear wavelets on Sierpinski gasket type fractals. J. Fourier Anal. Appl. 3 (1997), 387-416.

[22] Strichartz, R. S.: Function spaces on fractals. J. Funct. Anal. 198 (2003), no. 1, 43-83.

[23] Strichartz, R. S.: Mock Fourier series and transforms associated with certain Cantor measures. J. Anal. Math. 81 (2000), 209-238.

[24] Takens, F. and Verbitskiy, E.: On the variational principle for the topological entropy of certain non-compact sets. Ergodic Theory Dynam. Systems 23 (2003), no. 1, 317-348.

[25] Conze, J.-P. And Raugi, A.: Fonctions harmoniques pour un opérateur de transition et applications. Bull. Soc. Math. France 118 (1990), 273-310.

[26] Zygmund, A.: Trigonometric Series, vol. I. Cambridge University Press, London-New York, 1968.

Recibido: 17 de julio de 2003

Revisado: 20 de marzo de 2004

Dorin E. Dutkay

Department of Mathematics

Rutgers University

110 Frelinghuysen Road

Piscataway, NJ, 08854

ddutkay@math . rutgers . edu

Palle E. T. Jorgensen

Department of Mathematics

University of Iowa

14 MacLean Hall Iowa City, IA 52242-1419

jorgen@math.uiowa.edu

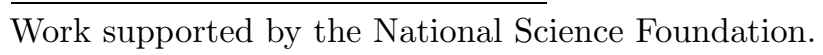

